\title{
Aerodynamics of a Supersonic Projectile in Proximity to a Solid Surface
}

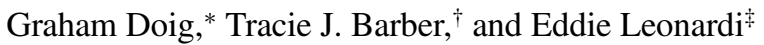 \\ University of New South Wales, Sydney, New South Wales 2052, Australia \\ and

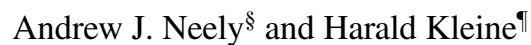 \\ University of New South Wales at the Australian Defence Force Academy, \\ Canberra, Australian Capital Territory 2600, Australia
}

\begin{abstract}
Flow around a Mach 2.4 NATO $5.56 \mathrm{~mm}$ projectile in close proximity to a ground plane was investigated using computational fluid dynamics for a direct numerical reproduction of live-range experiments. The numerical approach was validated against both the live-range tests and subsequent wind-tunnel experiments. A nonspinning half-model and a full, spinning projectile were examined to clarify the influence of rotation. Multiple ground clearances were tested to obtain clear trends in changes to the aerodynamic coefficients, and the three-dimensional propagation and reflection of the shock waves were considered in detail. The behavior of the flow in the near wake was also studied as ground clearance was reduced. Ground proximity was found to significantly increase the drag force acting on the projectile, as well as generate a force normal to the ground and an increased side force, when ground clearance was less than one diameter. For clearances between approximately 0.4 and 1 diameter, the pitching moment produced was nose-down. For lower clearances, a more distinct nose-up trend was produced. The generated side force was orders of magnitude lower than the normal and drag forces.
\end{abstract}

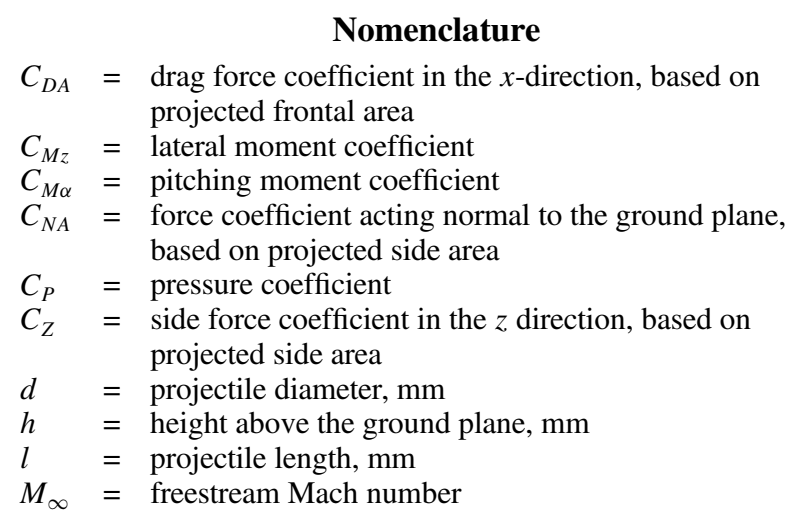

\section{Introduction}

A. Background and Motivation

LTHOUGH there is some evidence to suggest that researchers
have contemplated the effects of the flight of a projectile near a
wall or ground plane in the past [1], the aerodynamic consequences
of such a situation have not been formally addressed by the scientific
community despite relevance to, for example, urban combat
scenarios where bullets may be fired in close proximity to walls.
Shock reflections from the ground plane affect the flowfield around
the projectile; this degree of influence is dependent on the ground

*Research Associate, School of Mechanical and Manufacturing Engineering. Member AIAA.

${ }^{\dagger}$ Senior Lecturer, School of Mechanical and Manufacturing Engineering.

Professor, School of Mechanical and Manufacturing Engineering.

${ }^{\S}$ Senior Lecturer, School of Engineering and Information Technology. Senior Member AIAA.

${ }^{\top}$ Associate Professor, School of Engineering and Information Technology. clearance. The problem is unusual in that it involves a near-inviscid initial regular shock reflection at the ground plane, followed by both strong and weak shock/boundary-layer interactions in a strongly three-dimensional flowfield.

The presence of the ground leads to an asymmetry in the pressure distribution, which results in a force normal to the ground. The rotation of the projectile also introduces a second asymmetry in the pressure distribution when in close proximity to the ground, inducing a side force.

In a preliminary study devised at the University of New South Wales at the Australian Defence Force Academy [2], the flowfield around a standard NATO $5.56 \mathrm{~mm}$ round, fired from an ADI AUSSTEYR A1/A2 rifle and passing at Mach 2.4 over a ground plane at a range of clearances, was visualized by means of timeresolved schlieren using a high-speed video camera (Shimadzu HPV1). The video, captured at 500,000 frames per second, revealed how the shock waves between the bullet and the ground reflected and interacted with either the bullet or the wake or both.

The field of view and resolution of the schlieren visualization were not sufficient to determine any significant pitch or trajectory changes. Subsequent blowdown wind-tunnel tests lacked a force balance to directly measure the influence of the ground on the aerodynamic coefficients of the projectile. Therefore in order to fully investigate the influence of a ground plane on the projectile, these experiments were used as the basis for a comprehensive computational fluid dynamics (CFD) investigation.

The projectile, as shown in the schematic of Fig. 1, was composed of an ogive forebody, a narrow knurled strip to aid in the sealing of the projectile within the cartridge case, a near-cylindrical main body, which contacts the rifling of the barrel directly on firing, thus inducing spin, and a tapered boat tail blended to the flat, solid base. Grooved striations of approximately $7^{\circ}$ (produced by the barrel rifling) are present on the projectile main body in flight. Further details of the precise geometry can be found in the literature [2].

The rate of spin of the projectile under the test conditions described was determined from subsequent calculations, based on the barrel geometry and the striations measured on the projectile, to be $17,700 \mathrm{rad} \mathrm{s}^{-1}$ upon leaving the barrel [2]. The velocity of the projectile was $820 \mathrm{~ms}^{-1}$, corresponding to a Mach number of 2.4 and a Reynolds number of $1.056 \times 10^{6}$ based on the length of the 


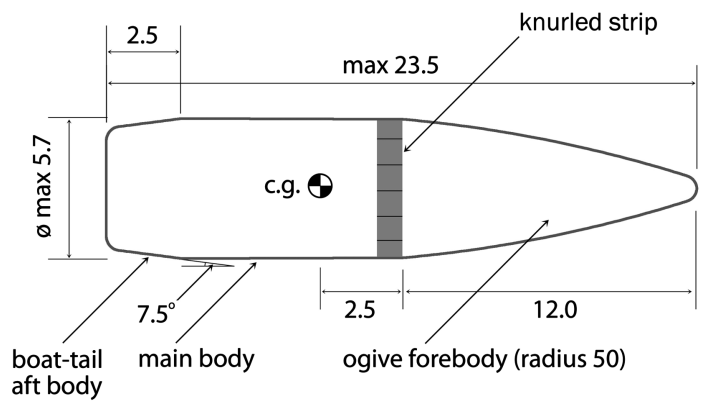

Fig. 1 Schematic of the projectile used for live-range experiments, dimensions in $\mathbf{m m}$.

projectile. Some salient features of the flowfield around the projectile in free flight are shown in Fig. 2, to aid the reader before the more detailed discussion later in the paper. In this image, one can observe the detached bow shock wave curving around the ogive forebody to form a gentle convex curve into the far field. Expansion waves generated along the ogive forebody follow this bow shock and eventually intersect with it, which weakens the shock by increasing its inclination. The knurled section at the transition from the ogive forebody to the cylindrical main body introduces a set of disturbances in the flow that continually evolve as the body spins. Approximately two projectile diameters downstream of the base, a recompression shock forms, serving to return the pressure in the flow field to approximately ambient conditions and to turn the flow back parallel to the path of the projectile. A wake pattern with clearly discernible turbulent structures is present in the separated region moving downstream of the boat tail, with "swirling" flow evident from the location of the recompression shock and downstream.

The ground effect flows that were observed in the live-range experiments fit three distinct categories as shown in Fig. 3. A type A interaction involves the reflection of the bow shock into the far wake, and the recompression wave trailing the projectile reflecting into the wake further still downstream. The overall influence on the aerodynamic forces and moments experienced by the projectile is negligible as the projectile moves with supersonic speed relative to the flow in the wake and is therefore not affected by disturbances in the wake. A type B case involves the bow wave reflection impinging on the near wake of the projectile (defined in this case as less than one projectile diameter from the base, which was observed to be the approximate maximum extent of recirculating flow for a free-flight case). The wake experiences a deflection due to the magnified pressure difference between the region below the wake and that above, and consequently the base pressure (and thus the drag) and, to a lesser extent, the projectile's pitching characteristics are affected. In a type $\mathrm{C}$ interaction, the bow wave reflects to impinge on the projectile body one or more times, and the recompression shock reflects into the wake to produce its own strong interaction. All aerodynamic forces and moments acting on the projectile are affected to varying extents by this scenario.

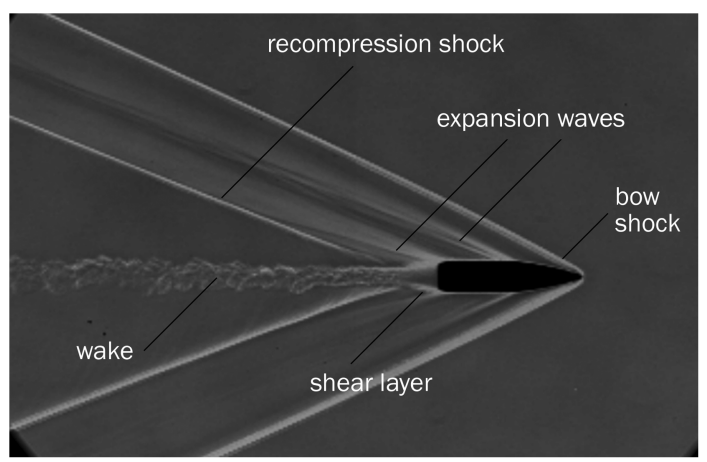

Fig. 2 Schlieren photograph with annotated flowfield characteristics for the projectile in free-flight conditions.

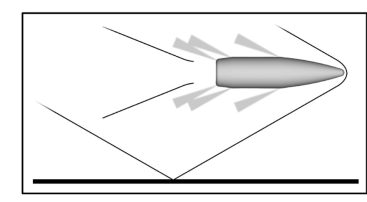

Type A

Shock reflection into

far wake, negligible influence on projectile.

a)

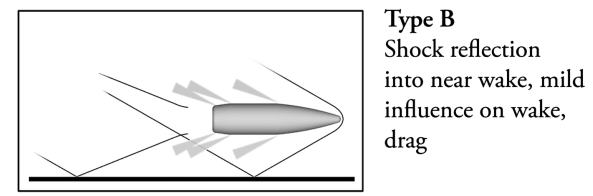

b)

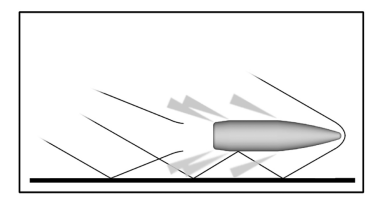

Type C

One or more shock

reflections onto projectile and into wake, influences all aerodynamic characteristics

c)

Fig. 3 Interpretations of observed shock reflection interaction scenarios, as categorized into three types.

Observations from experimental schlieren visualization of a type $\mathrm{C}$ case showed that the flowfield around the projectile as it moved over the leading edge of the ground plane was complex and initially transient, as the bow shock and the lower recompression shock reflect from the ground plane and interact with the projectile and the wake, respectively. The higher base pressure caused by the reflecting shocks leads to an adjustment of the angle of the upper recompression shock. This adjustment process is, however, finished within a distance equivalent to about three projectile lengths, and from then on the flow pattern is steady with respect to the moving projectile: all shocks and their reflections are highly consistent in time with respect to relative location. This established flowfield is suitable for analysis using steady-state numerical simulation and blowdown wind-tunnel experiments. Only the turbulent wake continues to develop over time along with mild wave interferences, produced by the knurled strip, which are not thought to affect the aerodynamic performance of the projectile in any irregular fashion.

A subsequent experimental program in a blowdown wind tunnel [3] used a symmetry method, which has been shown previously [4] to be an appropriate approximation of the moving ground that would normally be the correct way to address the ground boundary condition [5]. The test section had a cross section of $155 \times 90 \mathrm{~mm}$ and the projectile model was scaled to be 2.5 times the original size, with eight discrete pressure tappings on the surface of the projectile (six located axially on one side of the projectile, two on the diametrically opposite side). The Reynolds number of the experiment based on the model length was $1.31 \times 10^{6}$, which is 1.24 times the live-range Reynolds number, and thus within a reasonable margin of dynamic similarity for a flow regime where similarity in the Mach number is overwhelmingly the most important factor.

Both live-range and wind-tunnel test are used as qualitative and quantitative validation for the present numerical study, which uses Reynolds-averaged Navier-Stokes (RANS) CFD to thoroughly perform a detailed analysis of the flowfields obtained around the projectile at close ground proximity. The effect of the ground on the projectile is examined in terms of changes to forces and moments. The full three-dimensional flowfield is also investigated, with particular attention to the flow in the wake region and the way in which shock waves interact with the projectile and ground. In this respect, the computational modeling produces a wide range of information that could not be obtained from the two experimental programmes alone, and thus the three approaches combined produce a comprehensive body of knowledge with which to improve our understanding of these flow interactions. 


\section{B. Projectile Aerodynamics and CFD}

The use of RANS CFD for the investigation of projectile aerodynamics and flight has become relatively common amongst ballistics researchers, with a particularly large body of work produced by the U.S. Army Research Laboratory at their Aberdeen Proving Ground. Both in-house and commercial (CFD++ [6] , Fluent [7] ) codes have proven effective at predicting the aerodynamic characteristics of projectiles of varying complexity and dimensions.

A study using Fluent examined the effectiveness of turbulence modelling with regards to an axisymmetric wake region [7]. A fiveequation Reynolds stress model was chosen due to its higher-order properties, although no direct evidence was presented to support its advantages. Indeed, all the models tested were reported to perform similarly in terms of streamwise mean velocity in the wake. Mesh convergence studies were made for the wake region but did not address the resolution required to capture shock waves accurately away from the projectile surface.

The study by Silton of a full three-dimensional spinning projectile attempted to investigate the influence of geometric simplifications made in the interest of ease of modeling [6]. It was determined that ignoring the rifling grooves (striations) and other small-scale physical aspects of the body was not markedly detrimental to the prediction of aerodynamic forces and moments (backed by other experimental [8] and computational [9] studies drawing the same conclusion), although moments and derivatives directly related to the spinning motion of the projectile were not well predicted by the simplified model. A similar multiblock meshing strategy to the present investigation was used and showed good mesh convergence for grid sizes comparable to the standard meshes described in the following section, though no local mesh adaptation was investigated.

The study described in the present paper does not attempt to use the aerodynamic forces and moments predicted by computational modelling to undertake a full trajectory prediction analysis, although such a process has been proven as successful in comparison to experimental measurements $[10,11]$. That study showed that the motion of a spinning projectile at an angle of attack to the freestream depends heavily on the accurate prediction of damping derivatives and Magnus coefficients, though only the latter is considered in simple form in the present investigation in which all simulations were conducted for zero angle of attack. It is worth noting that the Magnus coefficient has been shown to be particularly sensitive to the periodic vortex shedding in the wake, though steady-state lift and drag force coefficients compare satisfactorily to experimental data when placed next to results from transient simulations [12].

\section{Supersonic Ground Effect}

Very few studies have been conducted on supersonic ground effect flows, and the applications have largely been related to the design of land speed record vehicles [13-15] or rocket-sled testing facilities $[16,17]$. Studies using rocket sleds are generally related to nonground effect applications, and the goal of researchers is to place the test object out of ground effect, though the rocket sled itself operates in such conditions. Several studies into the design of the Holloman facility in New Mexico were conducted during its life as a supersonic facility [16,17]. Wind-tunnel testing was conducted from $M=1.5$ to 4 , with an elevated ground plane. Rather than consistent trends, the force normal to the ground plane and the pitching moments acting on the model were observed to increase and decrease considerably across the Mach number range tested, indicating complex shock interactions from the simulated rail as well as the elevated ground plane, and downstream influences on the model sting and force balance due to multiple reflected shocks.

Although the flows observed in a supersonic ground effect regime are analogous to wind-tunnel wall interference scenarios, the concern of wind-tunnel testing is to avoid all forms of wall interference and in general a model would be situated as far as possible from any tunnel wall. In supersonic flows this is often relatively straightforward and reflected shocks would be unlikely to strike the body that originated them unless the fineness ratio was exceptionally large.
Studies of external stores on aircraft, which have in the past been investigated as simple supersonic streamlined bodies next to a solid surface [18], are also relevant to the present work. Generally though, more recent studies of stores consider cavities, on-wing configurations and other complex fully-viscous setups that produce flowfields that are far less applicable to the present study.

\section{Numerical Methods and Validation}

\section{A. Outline of Numerical Method}

A 64-bit commercial, finite-volume RANS solver (Fluent 6.3 [19]) was used to produce all results. A density-based, explicit coupled solver was combined with second-order upwinding to provide adequate steady-state solution accuracy. A multigrid technique was used to aid in the speed of solution, with each iterative step performed on four levels of progressively finer mesh.

The physical establishment of the wave reflections from the ground plane is a mechanically complex process, and thus significant instability was present in the solution during the early stages. This was overcome by incorporating a number of first-order iterations at a low Courant number until the shock reflection pattern began to form in the fluid, at which point second-order discretization was introduced. All cases were run until changes in all forces and moments acting on the projectile were negligible with continued iteration (less than $0.1 \%$ over 1000 continued iterative steps), implying that the flowfield near to the bullet was stable. A standard three-coefficient Sutherland viscosity model was used. The freestream density was set to match experimental conditions.

\section{B. Mesh and Boundary Considerations}

For all of the numerical analysis, the projectile was geometrically simplified by the removal of the knurled strip and rifling striations. This was an aid in mesh creation, as it eliminated the need to have an extremely fine mesh in these regions to resolve flow disturbances that are very weak relative to the major waves in the flowfield. It also meant that simulations did not have to be run as transient cases featuring a dynamic mesh. The simplified approach has been proven as effective at predicting aerodynamic characteristics of a similar projectile [6], though the dynamic derivative and roll damping coefficients are known to be more sensitive to the striations $[\underline{6}, \underline{8}]$. Because these derivatives and coefficients are not considered in the present study, the simplifications made are justified. All simulations were run for the projectile at an angle of attack of $0^{\circ}$.

In the simulations, a quarter-model was used for the free-flight nospin case, a half-model for the no-spin case with ground proximity, and a full model was used for all scenarios in which spinning is included. The frame of reference for the projectile is indicated in Fig. 4, and the computational domain for the simulation is depicted in Fig. 5 . Although the projectile's rate of spin will begin to decrease immediately upon leaving the barrel, it has been assumed in all



Fig. 4 Coordinate reference frame. 


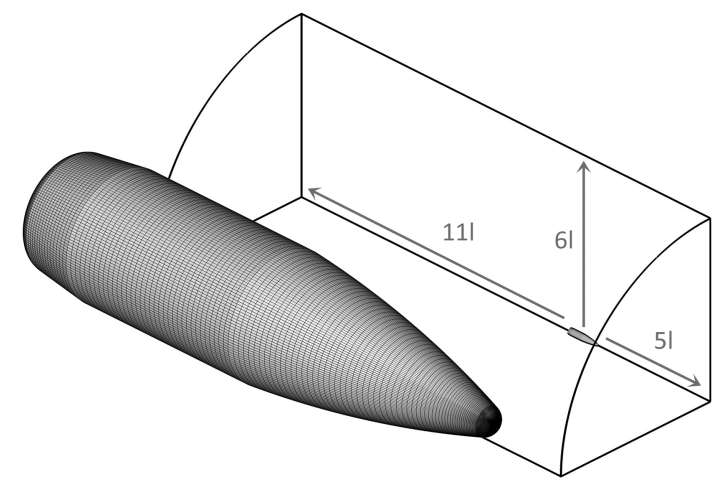

Fig. 5 Projectile surface mesh and example quarter-domain for liverange free-flight comparisons, with boundary locations normalized by projectile length, one.

simulations to be a constant $17,700 \mathrm{rad} \mathrm{s}^{-1}$ at all stages of motion. The rotation was applied as a simple boundary condition.

The sensitivity of the results with respect to the far-field boundary locations was examined by benchmarking forces obtained around the projectile in free-flight (quarter-model with symmetry) as shown in Table 1 for the standard mesh described next. Based on these results, a domain extending five projectile lengths upstream of the nose, 11 to the rear from the base, and six to the radial boundary was deemed acceptable, although it is acknowledged that all waves have become significantly diffused in the mesh by these boundaries.

Fully-structured multiblock meshes were generated in all cases. Extensive grid-refinement studies were conducted for a quarter bullet (free-flight with symmetry) model, then at several ground clearances for both spinning and nonspinning (half-model) projectiles. The meshes were similarly structured for each case, after the general approach depicted in Fig. 6. Over both the projectile surface and ground plane for all simulations, the wall $y^{+}$value was between one and two to facilitate effective resolution of the boundary layer, particularly in regions of shock interaction. Literature describing projectile research conducted using Fluent indicates that a $y^{+}$of less than one may even be preferable to predict the Magnus effect of a spinning shell [20], however, the present study is primarily concerned with the static force coefficients and the $y^{+}$of between one and two was deemed acceptable. The projectile surface mesh featured 160 circumferential cells, and this aspect of the mesh was not altered during the refinement studies.

Meshes of coarse, standard and finer density were constructed. The coarse mesh featured 130 lengthwise cells on the projectile surface, the standard: 255 , and the finer, 460 . This resulted in overall mesh sizes of $2.19 \times 10^{6}, 4.91 \times 10^{6}$, and $6.53 \times 10^{6}$ cells, respectively. Cells were clustered toward the projectile surface to resolve the boundary layer, and were more concentrated close to the nose, boat tail and base regions, where various waves originate.

If applied to simulations involving the full spinning projectile, the mesh was exactly doubled in size. The near-field mesh density in the wake and between the projectile and ground increased accordingly with the increases in bullet surface mesh cells, such that finer meshes had much greater resolution in the region underneath the body where the shock reflection patterns are most intense.

Table 1 Effect of variations in domain extent for a quarter projectile model

\begin{tabular}{ccc}
\hline \hline $\begin{array}{c}\text { Domain extent, normalized by } \\
\text { projectile length }(U=\text { upstream, } \\
D=\text { downstream, } R=\text { radial })\end{array}$ & Variation in $C_{N A}$ & Variation in $C_{D A}$ \\
\hline $3 U, 9 D, 4 R$ & $-2.92 \%$ & $-7.71 \%$ \\
$5 U, 11 D, 6 R$ (base case) & $-0 \%$ & $-\overline{0 \%}$ \\
$7 U, 11 D, 6 R$ & $0.02 \%$ & $0.05 \%$ \\
$5 U, 15 D, 6 R$ & $0.01 \%$ & $0.01 \%$ \\
$5 U, 11 D, 8 R$ &
\end{tabular}

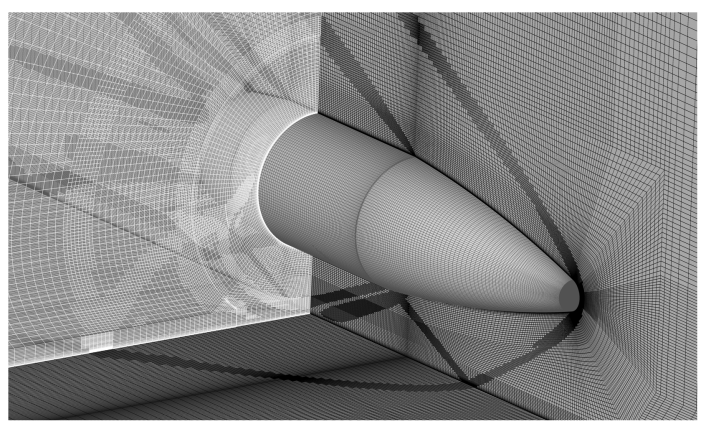

Fig. 6 Mesh on $x$-y plane (symmetry), projectile, volume slice, and "ground" plane (after shock-adaptation) for half-model (no-spin).

To capture the shock waves more effectively, particularly since they already suffer diffusion by virtue of numerous natural interactions with expansion waves, local mesh adaptation in regions of high local density gradient was performed. This was limited to no more than $3 \times 10^{5}$ cells per case (increasing the overall number of cells by a maximum $2.4 \times 10^{6}$ ). The local adaptation was performed on only standard and fine meshes.

The mesh upstream of the projectile, downstream in the wake, and to the radial far-field boundary, was nonconformal, with cells clustered closer to the projectile and growing slowly in volume from there as was indicated in Fig. 6 .

Detailed presentation of the full range of mesh sensitivity studies is not necessary to convey the outcomes; the case of $h / d=0.42$ (halfmodel, no-spin), in which the bow shock reflects to reimpinge on the projectile body, is representative of the approach applied to all cases. All results for this stage were generated using the Spalart-Allmaras (SA) turbulence model. Turbulence modelling is discussed in more detail in the following subsection.

In Fig. 7, one can see the influence of increasing mesh density on the resolution of the shock waves around the projectile. In this and subsequent figures, the levels depicting the contours of density across strong gradients have been exaggerated for clarity in grayscale depiction, and thus the shocks appear to be thicker than they are. The resolution of the mesh in the area between the projectile and the ground, in particular, was of interest in this scenario given the multiple reflections. Accompanying lift and drag results are presented in Fig. 8. The coarse mesh is able to capture the flowfield relatively well although the waves quickly diffuse into the far field, and a secondary reflection from the projectile to the ground is very weak and cannot sustain any subsequent reflection. The standard mesh presents an improvement, but the finer mesh shows that flow features in the wake and the multiple reflections between the projectile and the ground require this level of refinement to adequately resolve all the features of interest. Looking at the pressure distributions around the projectile for the $0 / 180^{\circ}$ plane, in Fig. 9 , further evidence of the improvements brought by refining the mesh can be observed. This ground clearance is unique in that the bow wave reflection impinges at the blend from the ogive to the main body, and thus the reflected shock and an expansion wave exist in very close proximity. The standard and coarse meshes have difficulties in clearly defining the flow in this region, whereas the fine and shockadapted meshes capture a distinct pressure spike at approximately $x / l=0.48$. A clearer visual indication of the beneficial influence of increased mesh density in this region can be seen in Fig. 10, where the resolution of the shock/boundary-layer interaction at the blend to the main body is critical. The base pressure profiles for the finer and adapted meshes are likewise similar, as opposed to the differing distributions predicted by the standard and coarse meshes.

As can be concluded from Fig. 8, the differences between all the meshes in terms of predicted forces are not substantial, with a difference in $C_{N A}$ from coarse to finest at a maximum of $2.5 \%$, and $C_{D A}$ a maximum of $6 \%$. The difference between fine and shockadapted standard meshes was modest in terms of drag but significant in terms of lift. Subsequent shock-adaptation of the finer mesh changed both values by less than $0.2 \%$. Therefore the standard mesh 


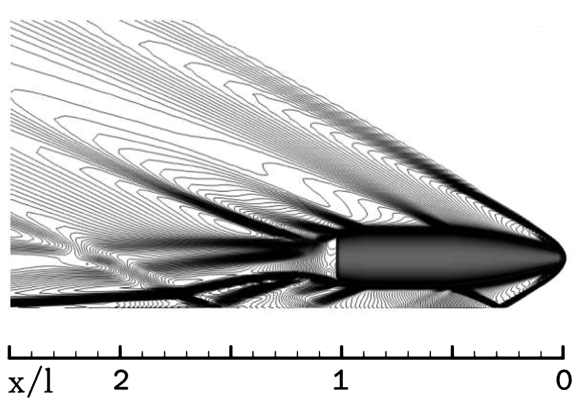

a)

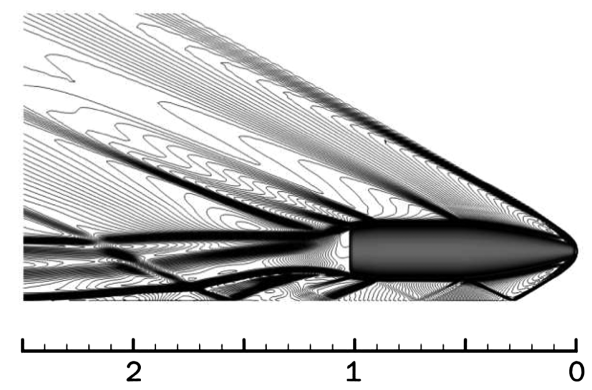

c)

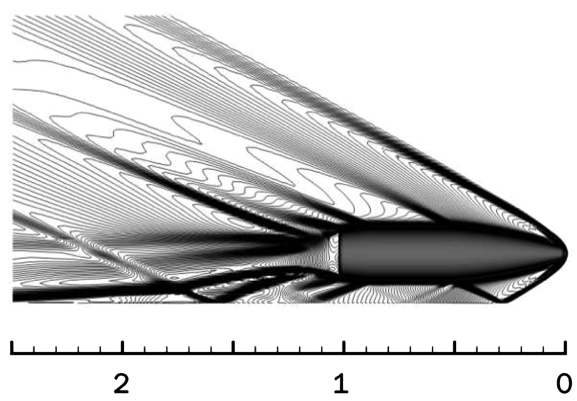

b)

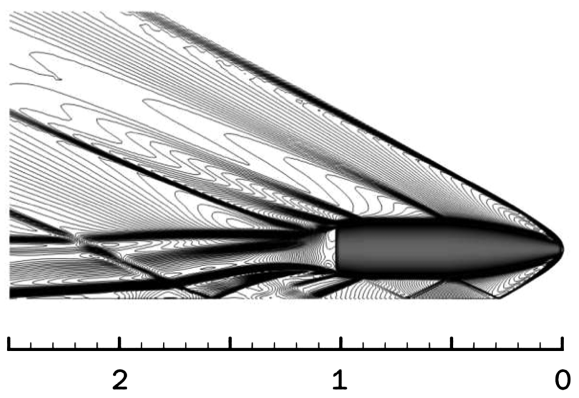

d)

Fig. 7 Contours of density as mesh is adapted for nonspinning (symmetry) bullet at $h / d=0.42:$ a) coarse $\left(2.18 \times 10^{6}\right.$ cells $)$, b) standard $\left(4.91 \times 10^{6}\right.$ cells), c) fine $\left(6.43 \times 10^{6}\right)$, and d) standard with shock-adaptation $\left(6.64 \times 10^{6}\right)$.

with shock-adaptation was adopted as the preferred method for capturing the waves as accurately as possible within sensible computational resource usage.

\section{Turbulence Modelling}

The flow in all simulations was assumed to be fully turbulent. The extent of any laminar flow on the model was not examined or fixed in experiments, however, most military projectiles feature a surface finish that enhances the transition to a turbulent boundary layer [21] and thus the assumption made with the computational model, in the absence of any special numerical surface treatment, is justified.

Selection of a turbulence model was based purely on the ability of tested models to reproduce the measured surface pressure distributions of the wind-tunnel model, given that no quantitative information about the wake or forces was available. Three models commonly used for aerospace problems were selected for this assessment: the one-equation SA model [22], the two-equation



Fig. $8 C_{N A}$ and $C_{D A}$ coefficients as half-model (symmetry) mesh is refined: a) coarse, b) standard, c) fine, d) standard with shockadaptation, and e) fine with shock-adaptation. realizable $k-\varepsilon$ model [23], and Menter's two-equation $k-\omega$ shearstress transport (SST) model [24]. It must be remembered that twoequation models have varying degrees of viscous dissipation, which is an important consideration in the presence of multiple shock reflections.

Figure 11 shows the $C_{P}$ predictions, for the $0^{\circ} / 180^{\circ}$ plane, of all three models for a wind-tunnel test at $h / d=0.5$. They all capture the flowfield adequately, with the realizable $k-\varepsilon$ predicting an earlier shock impingement on the projectile at approximately $x / l=0.57$, as well as a noticeably different pressure distribution toward the rear of the boat tail section. The error bars reported in Fig. 11 are based on the scatter of results obtained over multiple tests. It is worth noting that in all comparisons to experimental data, the CFD predictions of pressure distribution are consistently approximately 0.02 to 0.03 higher. This difference exists irrespective of mesh or turbulence model choice and in absolute terms the offset is around $1 \mathrm{kPa}$.

Of interest is the spike in the pressure distribution close to $x / l=0.02$. This would appear to be a mild compression or weak shock wave caused by an overexpansion of the flow at the shoulder of the small-radius nose [25], and is barely predicted by the realizable $k-\varepsilon$. Shock-adaptation of the mesh for the realizable simulations does not extensively change this. Since the compression lies upstream of the first pressure tapping, and all the models predict similar $C p$ at that point, it is difficult to determine objectively which model is predicting the flow more accurately. As it is, the only clear basis for making an informed choice is the performance along the $0^{\circ}$ plane, where the SA and SST models produce results slightly closer to experimental values. For reasons of reduced viscous dissipation across shock waves and reduced computational expense, the oneequation SA model was selected for all subsequent simulations.

One final useful comparison of the one-equation model to available experimental output with this model can be seen in Fig. 12, in which the standoff distance of the bow shock observed from a close-up schlieren image is shown, with CFD contours of Mach number overlaid. In this experiment, the bullet surface was etched with a sequence of grooves of $0.2 \mathrm{~mm}$ depth. At any location close to the surface, where the local Mach number exceeds unity, such a groove generates a weak Mach wave. The extent of the subsonic zone then follows from determining the position of the first of these weak waves. 


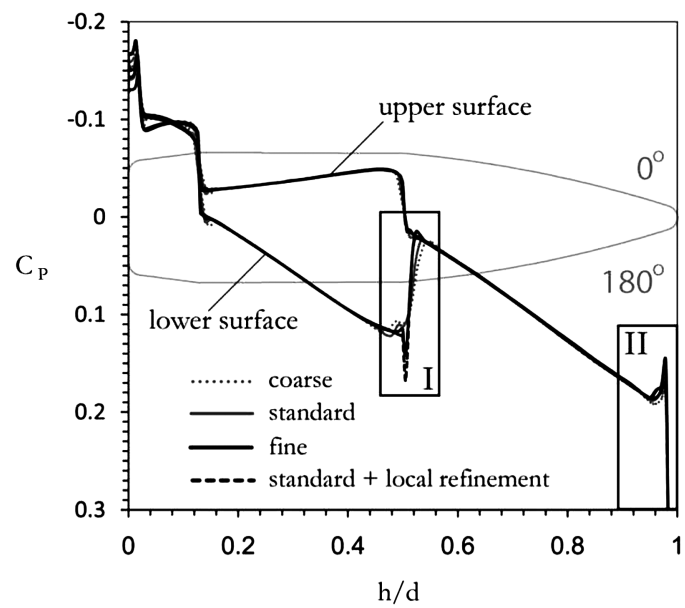

a)
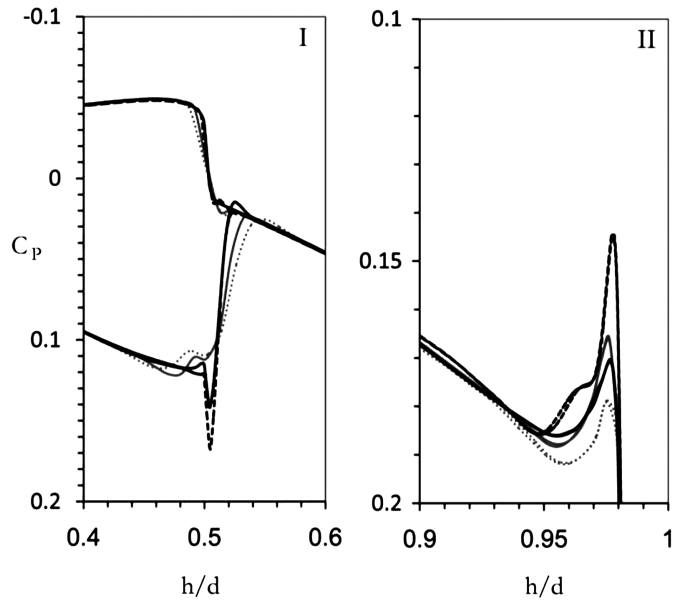

b)

Fig. 9 a) Pressure coefficients over the bullet at $h / d=0.42$ on 0 and $180^{\circ}$ planes as mesh is refined: a) regions of interest $I$ and $I I$ and b) detailed pressure distributions in I and II.
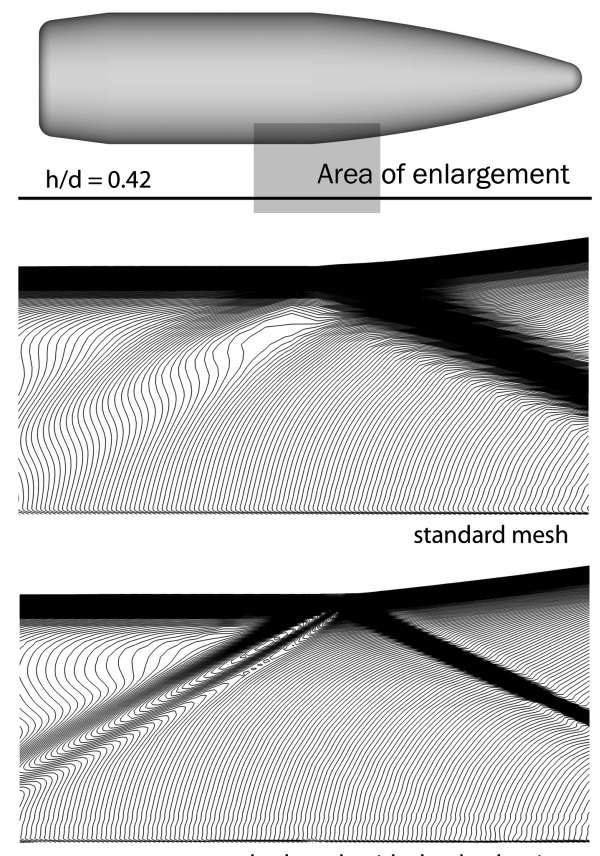

standard mesh with shock adaption

Fig. 10 Example of beneficial shock-adaptation for the case of $h / d=0.42$, illustrated by contours of density.

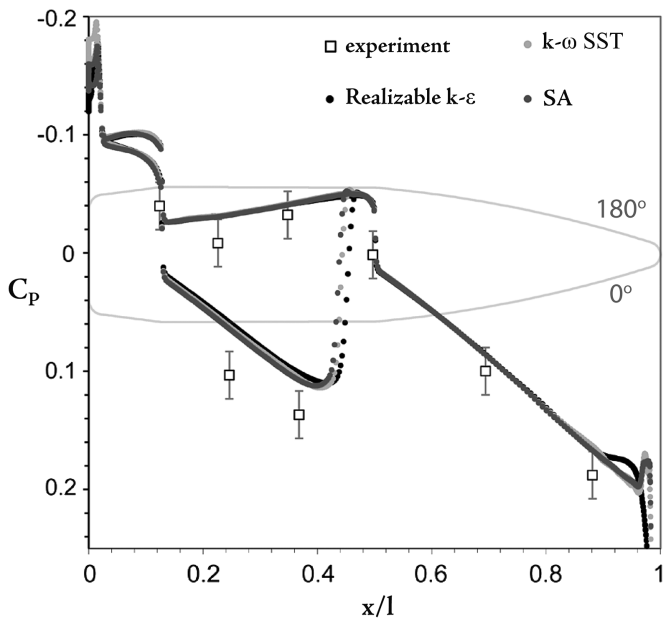

Fig. 11 Turbulence model comparisons for pressure coefficient distribution around the wind-tunnel model for the $0 / 180^{\circ}$ plane.

The bright white area along the bullet axis is a reflection of the flash lamp used for frontal illumination of the model. The extent of the subsonic zone around the tip was estimated with considerable accuracy by the CFD, as was the standoff distance of the shock provided the mesh was sufficiently fine (i.e. following shockadaptation).

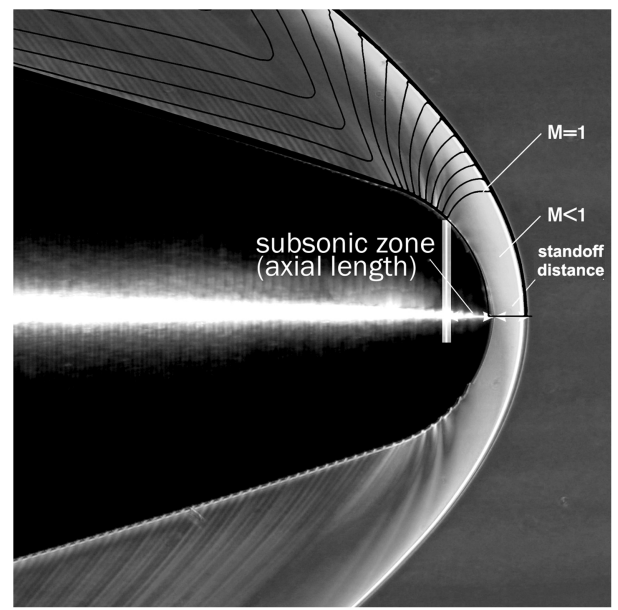

Fig. 12 CFD to wind-tunnel model comparison for shock standoff distance and location of reestablishment of supersonic flow.

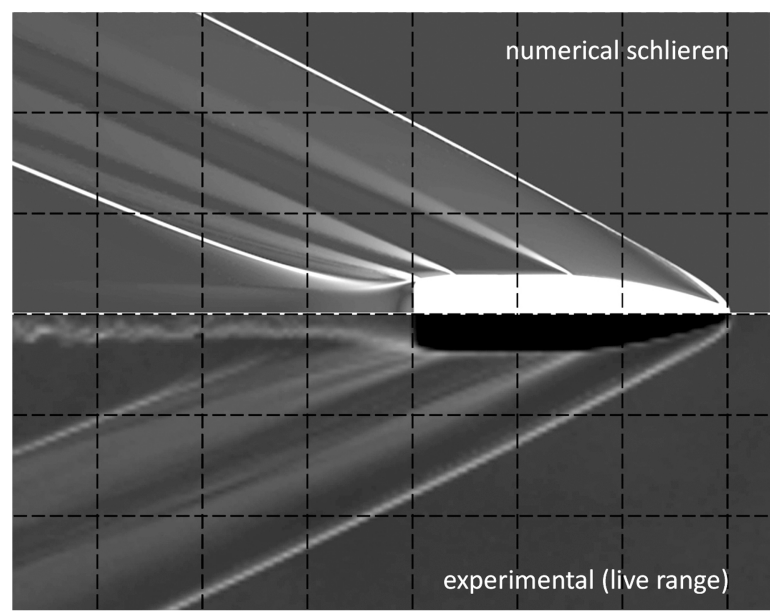

Fig. 13 Numerical schlieren comparison to an instantaneous schlieren image from time-resolved live-range footage of the free-flight (no ground) case. 
Table 2 CFD cases for live-range comparisons

\begin{tabular}{ccc}
\hline \hline$h / d$ & $\begin{array}{c}\text { Mesh cells } \\
\text { (after adaptation) }\end{array}$ & $\begin{array}{c}\text { Reflection/interaction } \\
\text { type }\end{array}$ \\
\hline $\begin{array}{c}\text { Free flight } \\
2.17\end{array}$ & $15.7 \times 10^{6}$ & - \\
1.77 & $17.5 \times 10^{6}$ & $\mathrm{~A}$ \\
1.31 & $14.4 \times 10^{6}$ & $\mathrm{~A}$ \\
1 & $13.8 \times 10^{6}$ & $\mathrm{~B}$ \\
0.75 & $13.2 \times 10^{6}$ & $\mathrm{~B}$ \\
0.5 & $12.6 \times 10^{6}$ & $\mathrm{C}$ \\
0.42 & $12.2 \times 10^{6}$ & $\mathrm{C}$ \\
0.365 & $12.1 \times 10^{6}$ & $\mathrm{C}$ \\
0.2 & $11.8 \times 10^{6}$ & $\mathrm{C}$ \\
\hline
\end{tabular}

\section{Comparison with Live-Range Schlieren}

A similar comparison to the live-range schlieren is shown in Fig. 13, highlighting good agreement in terms of shock angles and wake thickness. Table 2 outlines the CFD cases for simulations of the projectile under the live-range conditions. All ground clearances were examined for both a spinning and nonspinning (half-model) projectile.

Ground clearances of $h / d=2.17,1.77,1.31$ and 0.75 equate directly to experiments, for which numerical schlieren comparisons to instantaneous stills from the experimental video are presented in Fig. 14. Qualitatively, the images show good agreement for the shock locations and behavior in reflecting from the ground plane. At $h / d=2.17$ and 1.77 , which represent type A reflection interaction cases, the bow wave reflecting from the ground plane passes relatively unaffected through the recompression shock and interacts with the wake several projectile diameters downstream of the projectile base. The wake thickens slightly but the wave passes largely unaffected through it, interacting with the recompression shock in the far field.

At $h / d=1.31$, which is of type $\mathrm{B}$, the bow wave reflection interacts strongly with the near wake and does not reach the far field with any significant strength. The wake itself is deflected upwards and the formation of the recompression shock is affected, resulting in a slightly adjusted shock angle as viewed on this plane. The reflection of the recompression wave itself causes a noticeable downwards deflection in the wake far downstream of the projectile base.

At $h / d=0.75$, a type $C$ reflection interaction occurs, with the bow shock reflecting onto the projectile, impinging on the main body to the rear of the c.g., resulting in a subsequent secondary reflection from the projectile to the ground, which itself reflects into the wake region. At this point it largely dissipates, and the wake is drawn markedly toward the ground. As the pressure distribution changes over the projectile, the recompression shock is altered, with the region of the wave in closest proximity to the ground turned toward the ground plane, and reflecting into the wake to thicken it further while at the same time turning it back toward a streamwise alignment.

In all cases, the angle of the recompression shock is also strongly influenced by the way in which the shear layer leaves the base of the projectile and forms the general outline of the wake region-the disintegration of the shear layer into a turbulent structure at approximately $1 d$ from the base is a purely transient phenomenon not well represented in the steady-state simulations. However, when
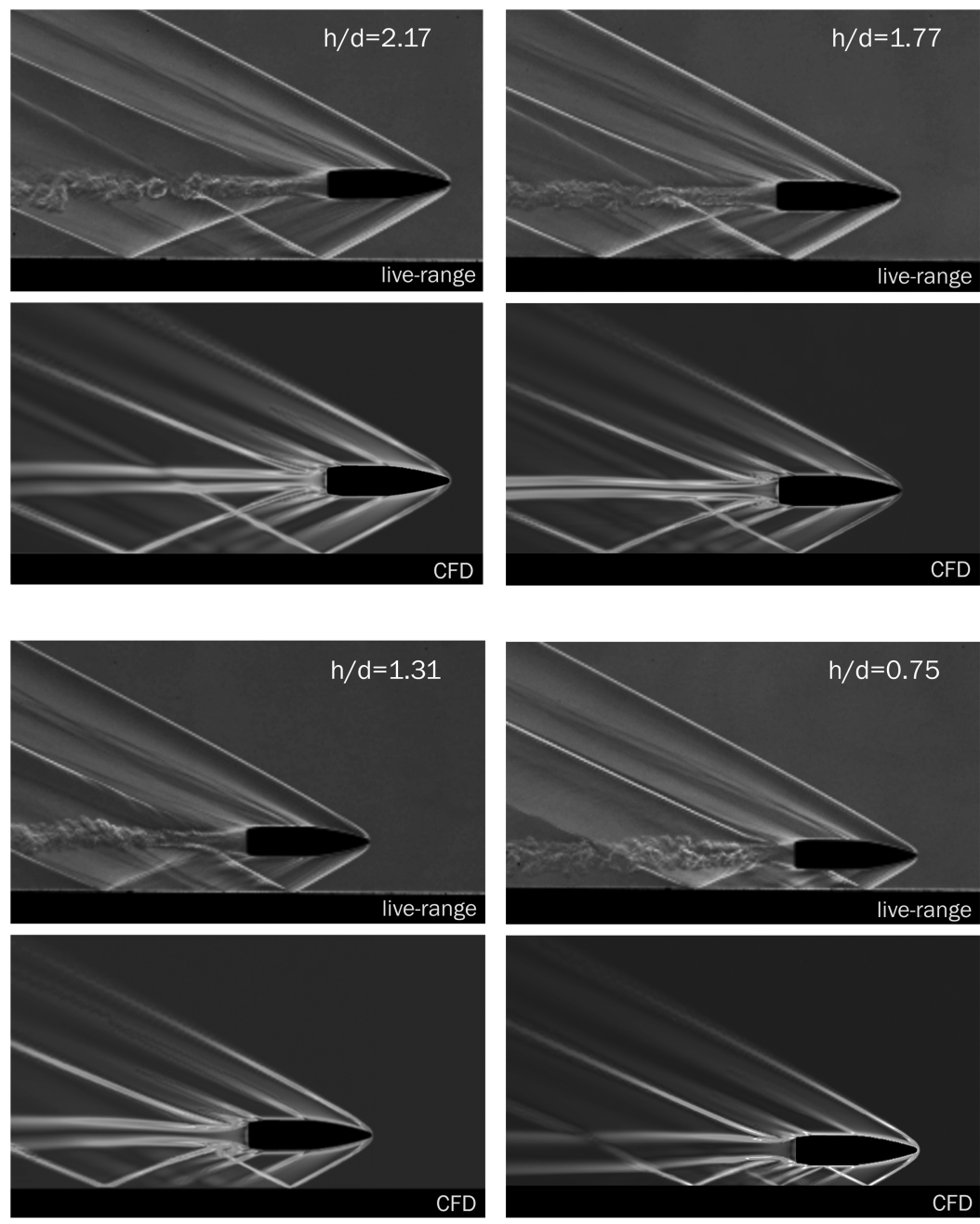

Fig. 14 Instantaneous schlieren images from time-resolved live-range footage with numerical schlieren comparisons showing good agreement with regards to shock angles and wake disturbances. 

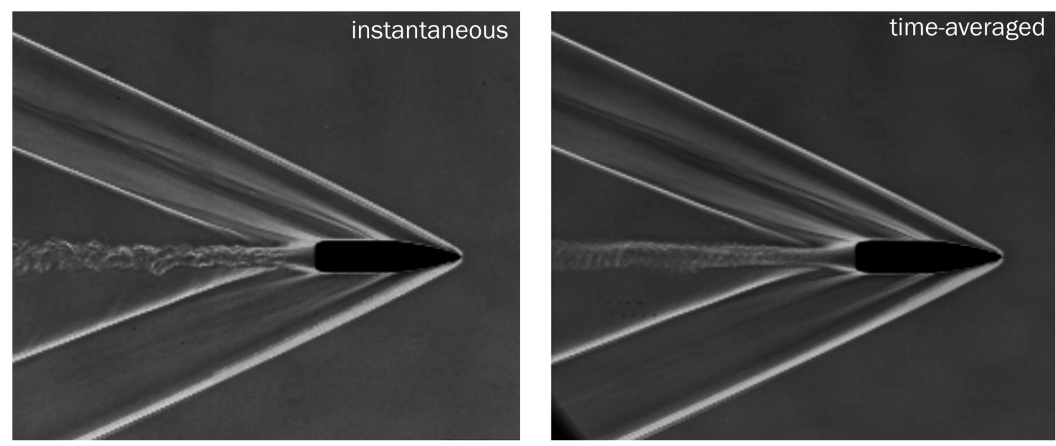

Fig. 15 Instantaneous vs time-averaged schlieren images from the live-range experiments.
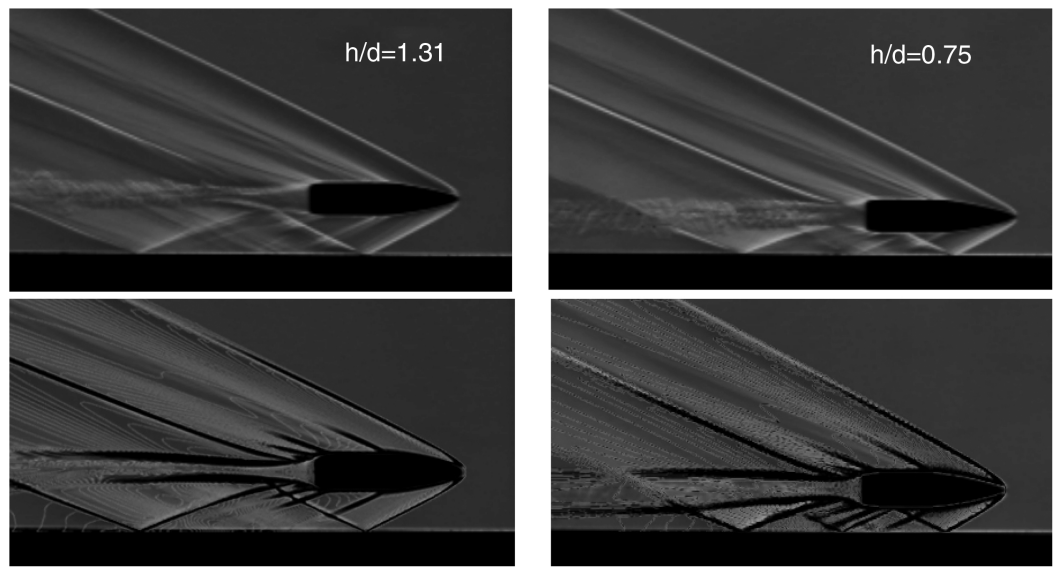

Fig. 16 Time-averaged (over $5 \times 10-5$ s) schlieren images from the live-range video footage (top) for $h / d=1.31$ and $h / d=0.75$ with CFD contours of density overlaid (bottom).

approximately 30 still images from the experiments are manually overlaid frame by frame, a pseudosteady flowfield more akin to the CFD begins to emerge, further highlighting the steady nature of the shock waves around the projectile. The choice of 30 images was determined purely by the number of frames that could be used while still preserving a reasonable field of view of the flowfield, and more would have been preferable. Using this technique in Fig. 15, the extent of the shear layer and wake is considerably clearer than in the instantaneous still, and thus a more appropriate comparison to the numerical modelling is possible; this technique has been used to create the comparisons between CFD and experiment in Fig. 16, in which excellent agreement between shock angles and locations is shown. Again, these figures contrast a type $\mathrm{B}$ and type $\mathrm{C}$ interaction, whereby the wake of the $h / d=1.31$ case is observed to experience a slight upwards deflection as the first reflection of the bow shock interacts with the flow behind the projectile base. At $h / d=0.75$, the wake features a noticeable downwards deflection. We will return to consideration of the wake later, where the mechanisms at work are discussed in more detail.

The lowest ground clearance observed in the experiments was $h / d=0.75$, and for closer ground proximity, only the numerical analysis is able to provide data.

\section{Numerical Results for Spinning Projectile}

\section{A. Three-Dimensional Flowfield}

In the images presented to this point, the flow has largely been examined on the $x-y$ plane, perpendicular to the ground. For ground effect cases, however, it can be misleading to ignore the strongly three-dimensional effects of the shock reflections. The waves that reflect from the ground and impinge upon the projectile do not simply reflect at an angle consistent with oblique shock/boundary-layer interaction theory: this only holds for the wave as it exists purely on the $0^{\circ} / 180^{\circ}(z=0)$ plane, or the symmetry plane for the half-model

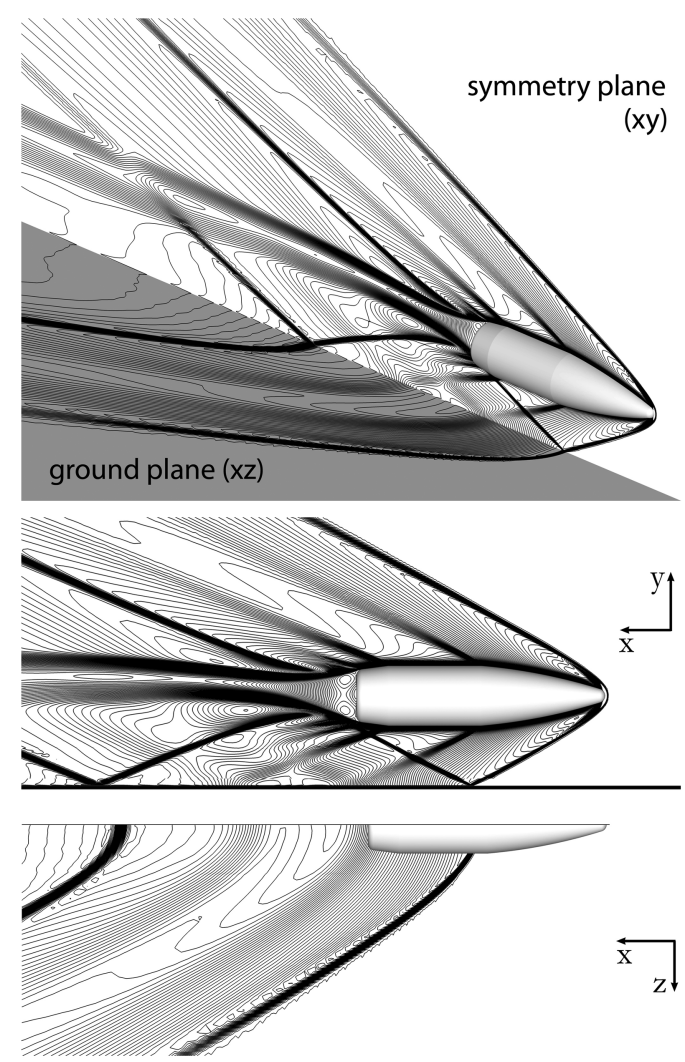

Fig. 17 Density contours on the symmetry and ground planes for nonspinning projectile at $h / d=1$. 


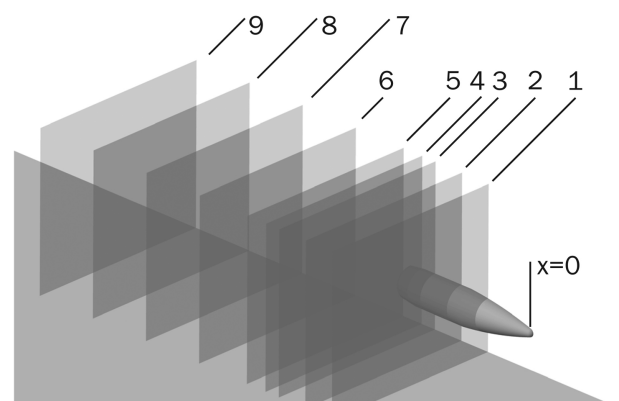

Fig. 18 Slice locations from projectile nose: 1) $1.49 d$, 2) $2.37 d, 3) 3.25 d$, 4) $3.68 d(1 \mathrm{~mm}$ downstream of the base), 5) $4.30 d$, 6) $5.88 d$, 7) $7.63 d$, 8) 9.39d, and 9) 11.14d.

simulations. All results in this section are drawn from simulations involving the spinning projectile at zero angle of attack.

Figure 17 presents density contours on the symmetry and ground plane around the projectile at an example ground clearance of $h / d=1$. Figure 18 shows a series of slices through the flowfield taken perpendicular to the $0^{\circ} / 180^{\circ}(z=0)$ plane, which are used to examine the three-dimensionality of the flowfield in Fig. 19, in which numerical schlieren on eight of the nine slices taken through the flowfield is shown.

Slice 2 ( $2.37 d$ from the nose of the projectile) presents the initial reflection of the bow wave from the ground plane as the rest of the wave propagates conically outwards. At this point an extremely thin boundary layer begins to form on the ground as a result of the impinging bow shock and its induced flow.

Slice $3(3.25 d)$ shows the reflected bow wave passing through the expansion from the blend onto the main body. Slice 4 (4.30d) shows the reflected bow wave interacting with the near wake, $1 \mathrm{~mm}$ behind the projectile base, as the trailing expansion wave fans out. Also of note is the triple-point structure that the front of the bow wave now forms close to the ground. As it propagates into the far field, the wave front now incorporates a clear Mach stem at the ground, which increases in extent as the structure evolves downstream.

The shock interaction with the wake in which the flow is recirculating involves the wave being weakly reflected in coincidence with the emerging recompression shock, rather than simply passing through the wake region. The remainder of the wave refracts around the wake core as shown in slice 5 , with the pressure difference below
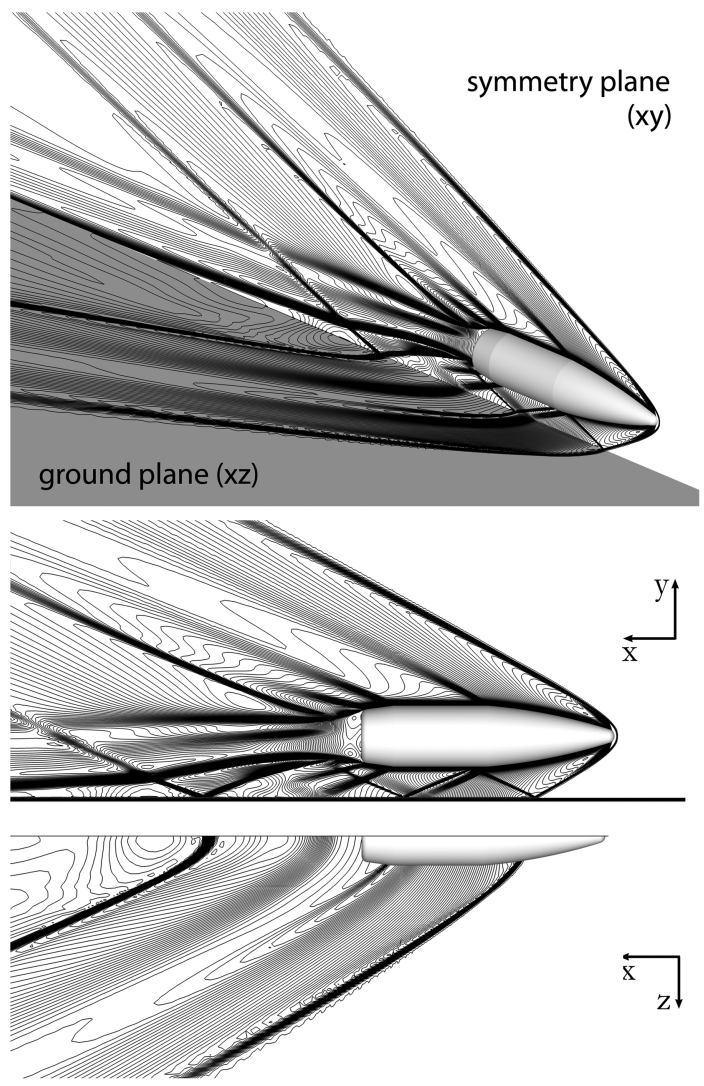

Fig. 20 Density contours on the symmetry and ground planes for nonspinning projectile at $h / d=0.5$.

and above the wake being great enough to draw flow upwards, resulting in the deflection of the wake seen previously in images of type B cases. The wake is also clearly distorted by the shock interaction, such that the shape of the recompression shock is highly asymmetric above and below the core of the wake. The remainder of the bow reflection circumnavigates the perimeter of the recompression shock into slice 6 , continually weakened by the expansions stemming from the boat tail.

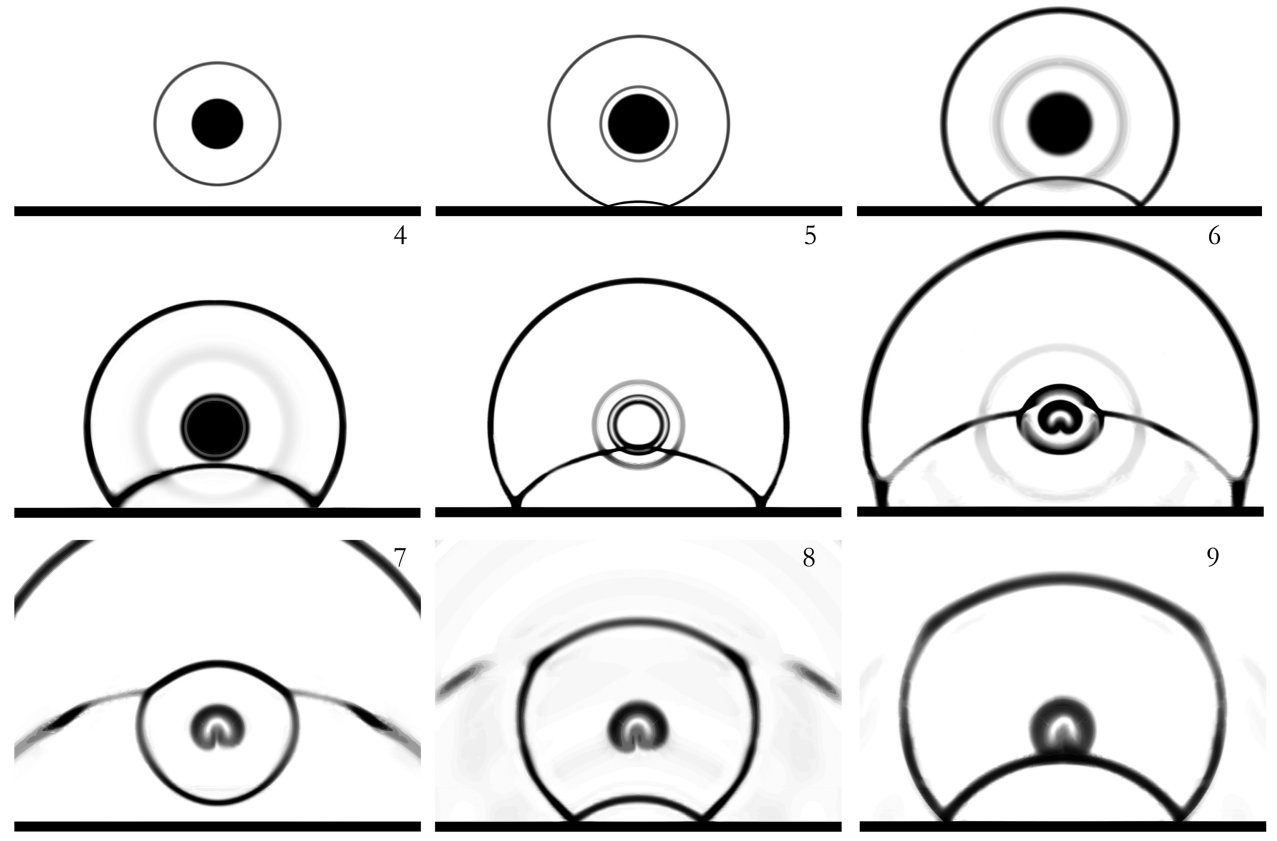

Fig. 19 Numerical schlieren for flowfield around nonspinning projectile at $h / d=1$, for slice locations described in Fig. 18. 


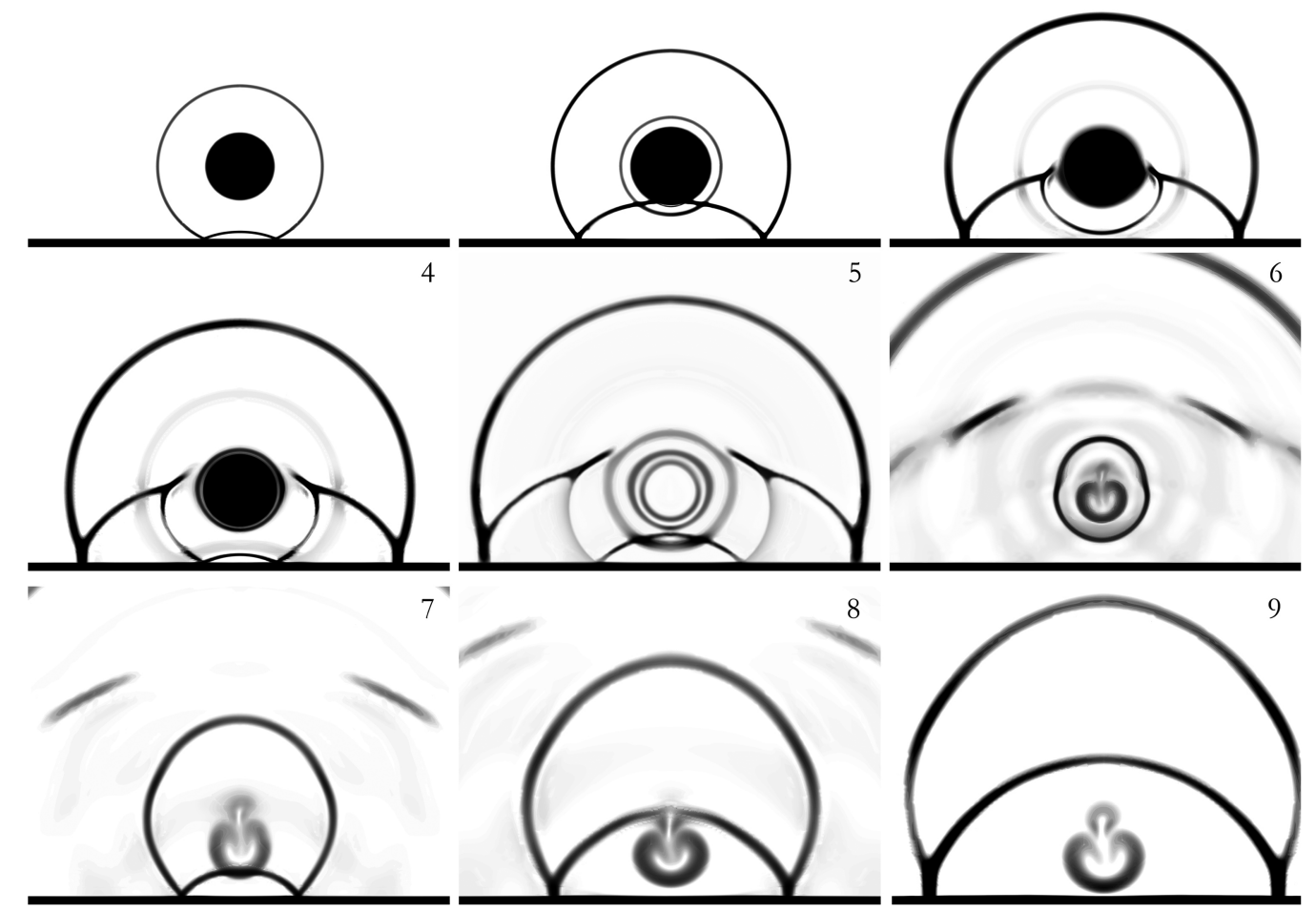

Fig. 21 Numerical schlieren for flowfield around nonspinning projectile at $h / d=0.5$, for slice locations described in Fig. $\underline{18}$.

In slice $8,9.39 d$ downstream from the nose of the projectile, the recompression shock has experienced its own reflection from the ground plane, and because there now exists a very thin boundary layer, a weak viscous interaction takes place, and the wake vortices have reached their maximum height from the ground. In slice 9 the reflected recompression shock begins its wake interaction.

Density contours and slices for a type $\mathrm{C} h / d=0.5$ are presented in Figs. 20 and 21 . At this lower clearance, the bow wave reflects from the ground in slice 1, and the reflection impinges on the projectile in slice 2 slightly downstream from the blend from the ogive section to the main body, which produces the expansion wave. In slice 3 the reflection propagates around the projectile circumference, featuring a distinct triple point. The front of the wave is perturbed by the projectile body, giving it a significant component in the $x-y$ plane. The boundary layer is influenced by the perpendicular Mach stem at the body formed from the initial reflection, drawing fluid upwards around the projectile and causing a flow asymmetry between the

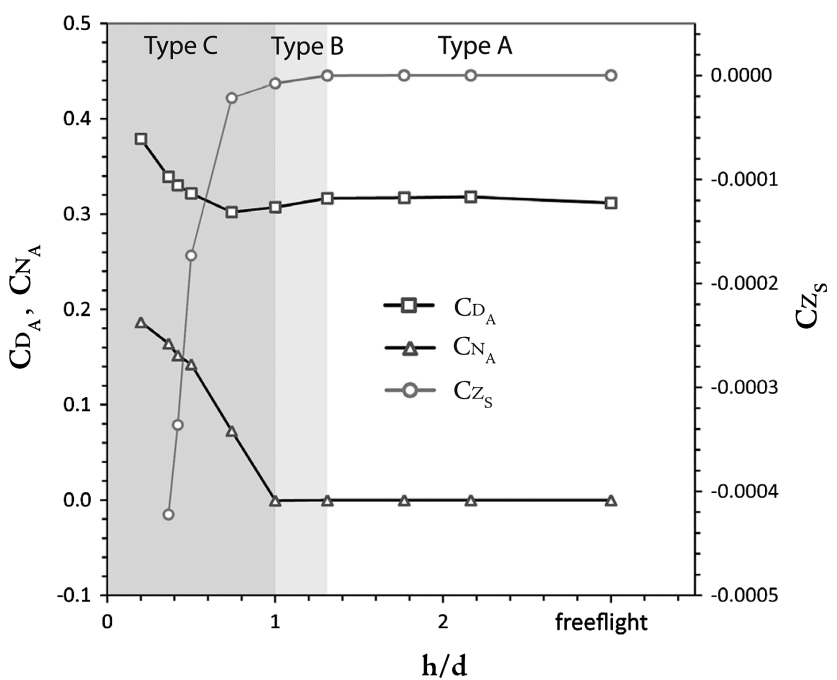

Fig. 22 Variation in force coefficients for spinning projectile as ground clearance is reduced. upper and lower halves of the body, which is more clearly seen in slice 4.

Slice 4 also shows the front of the reflected bow wave beginning to propagate away from the surface of the projectile, no longer able to follow the curvature of the body. This is accompanied by the expansion waves from the transition to the boat tail region, and slice $5,1 \mathrm{~mm}$ behind the base of the projectile, indicates that these expansions and the last vestiges of the original reflection accompany each other into the far field. The secondary reflection of the bow wave from the ground now impinges upon the free shear layer, but interacts strongly with the expansions from the base region and is significantly weakened before any meaningful wake interaction can occur.

In slice 6 , the effect of the asymmetric pressure distribution and boundary-layer thickness around the projectile clearly manifests itself in the formation of the recompression shock, which is distinctly asymmetric, and a strong downwards suction of flow in the center of the wake is observed. Thus the wake is drawn toward the ground in the subsequent slices, initially elongating vertically in slice 7 , then

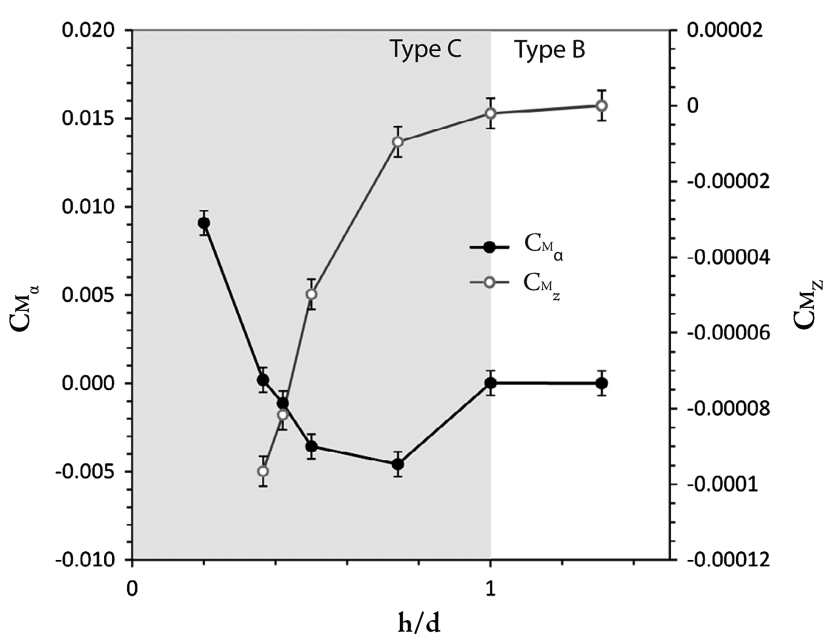

Fig. 23 Variation in moment coefficients for spinning projectile as ground clearance is reduced. 
fattening again as the reflection of the recompression wave passes through it. By slice 9, the wake vortices are beginning to interact with the ground plane itself, and will continue to expand laterally in the presence of this boundary.

The flow patterns and images obtained are reminiscent of those produced by Marconi (Oberoi et al. [14]) for an inviscid simulation (validated against experimental results) of a store/plate interaction. The transition of the propagating bow wave reflection at the ground plane from regular to Mach reflection, noted in Marconi's work, can also be observed in Figs. 19 and 21. It is a consequence of the fact that the bow shock expands radially so that at some distance from its origin it will form a Mach reflection, similar to a blast wave generated above ground. This process is independent of the forward motion of the projectile and the resulting relative velocity between projectile and ground.
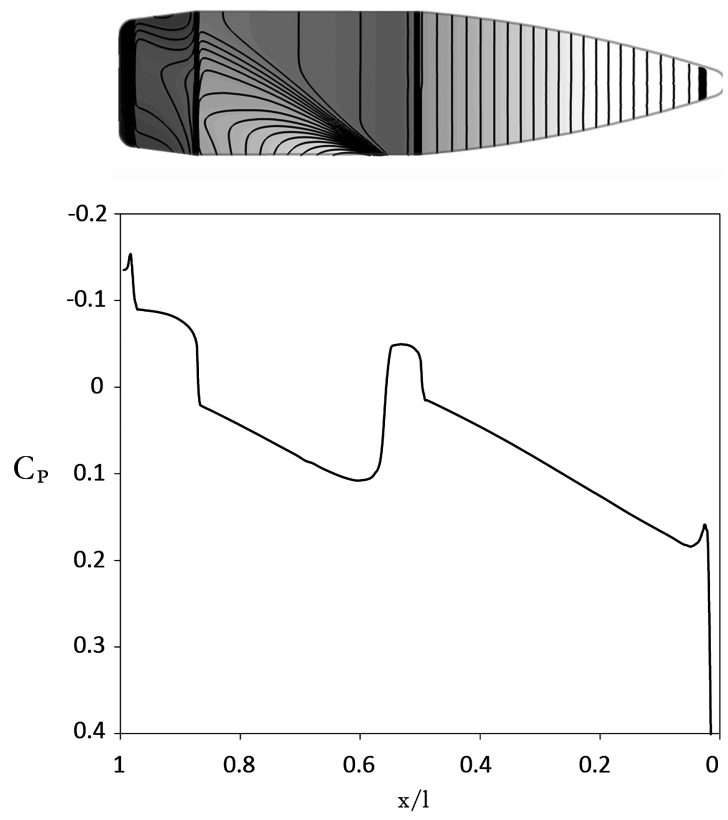

a) $\mathbf{h} / \mathrm{d}=\mathbf{0 . 5}$
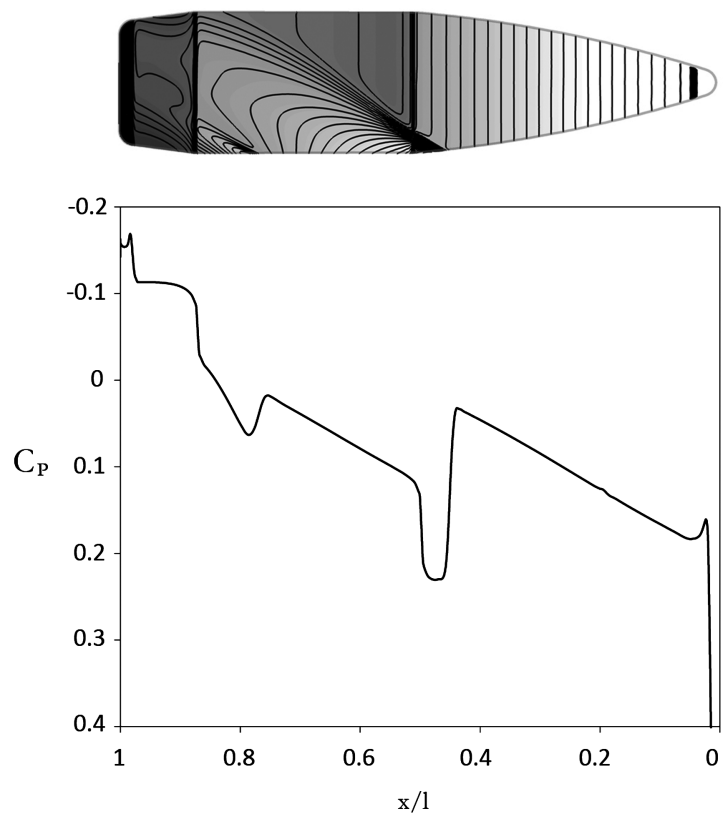

c) $\mathbf{h} / \mathbf{d}=\mathbf{0 . 3 6 5}$

\section{B. Force and Moment Coefficients}

Figures 22 and 23 present predicted aerodynamic force and moment coefficients for the full spinning projectile. The force coefficients for the nonspinning projectile are not shown, but for comparison they were found to be close to identical across the range of ground clearances, with a maximum difference in both lift and drag coefficients of less than $1 \%$ even at low ground clearances.

The drag coefficient is almost constant for the type A reflection interactions, and dips slightly in the region of type B cases. This seems to indicate that the influence of the strong reflection of the bow wave on the near-wake region reduces the extent or strength of the wake and recompression shock.

At clearances lower than $h / d=0.75$, the drag increases markedly. Despite the shock impingements on the projectile, the viscous drag on the body does not change by anything more than a few percent; the
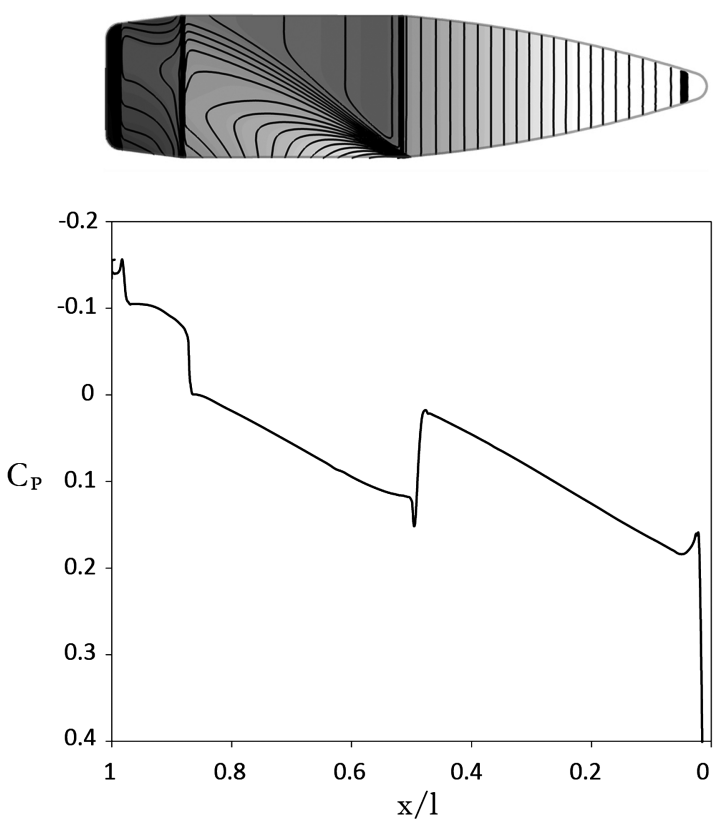

b) $h / d=0.42$
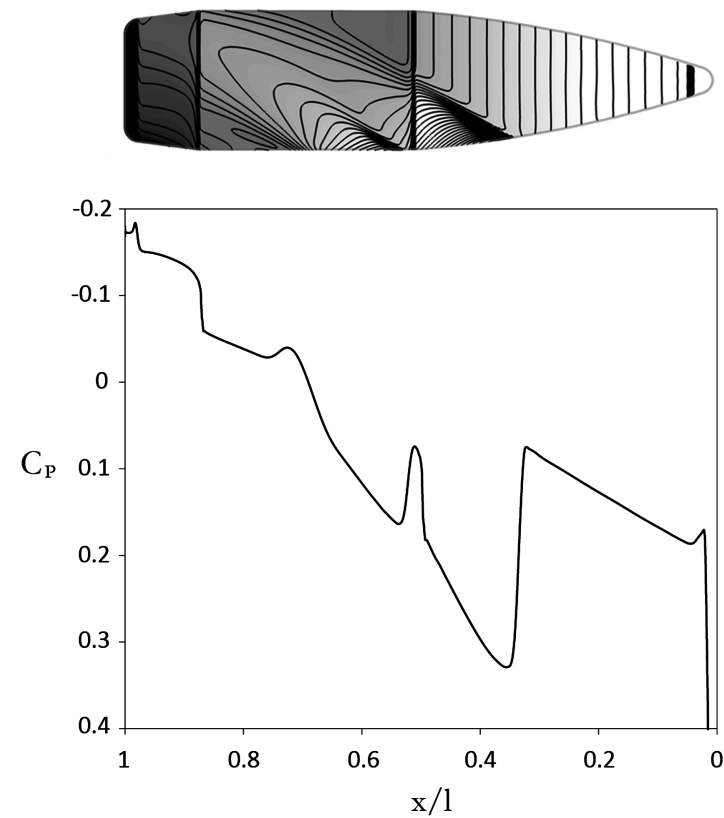

d) $\mathbf{h} / \mathrm{d}=\mathbf{0 . 2}$

Fig. 24 Pressure distributions on the lower surface at the midplane $(z=0)$ for projectile at ground clearances of a) $h / d=0.5$, b) $h / d=0.42$, c) $h / d=0.365$, and d) $h / d=0.2$. 
main driver for the increased total drag is the effect the reflections have on the base pressure and therefore the wake. As the wake thickens and is distorted, it deflects increasingly toward the ground and drag rises accordingly.

The force normal to the ground plane that acts on the projectile remains insignificant for type $\mathrm{A}$ and type $\mathrm{B}$ reflection interactions. However, an increase of several orders of magnitude is observed for type $\mathrm{C}$ cases, in which the pressure distribution around the projectile is directly affected. Given that the pressure downstream of a shock is higher than that ahead of it, the normal force increases in direct relation to the amount of high pressure produced on the lower half of the body surface. As the projectile is spinning, this normal force would not produce lift directly, but rather result in a perpendicular precession causing lateral movement. Therefore the main influence of ground proximity would not be to induce significant movement toward or away from the ground plane, but rather a horizontal travel.

To place the increase in normal force in perspective, consider that while the projectile itself has a downwards force due to gravity equivalent to approximately $0.185 \mathrm{~N}$, the normal force acting on it at the most extreme $h / d$ examined, 0.2 , is $1.956 \mathrm{~N}$. This normal force coupled with the rotation of the projectile induces a precession and hence a motion to the side. This effect is enhanced when the ground clearance is further reduced due to gravity. In the case of a finite ground plane, as in the original experiment, the overall deviation, however, would be small as the influence of the ground would be short-lived. In this respect, the side force of $-0.0189 \mathrm{~N}$, generated by the asymmetry of the pressure distribution, which is caused by the rotation of the projectile in the presence of the ground, could also be a significant contribution (around 10\% of the force of gravity). This force would result in a tendency for vertical movement relative to the wall once again due to precession of the rotating projectile.

The motion of the projectile would not simply be a result of the normal, side and drag forces, however. The pitching tendency of the body is affected by the shock reflections as shown in Fig. 23, as referenced to the projectile's center of gravity, which was measured to be at $x / l=0.64$ from the nose of the projectile in a simple balance test. As a result of the measurement technique, error bars have been incorporated into the graph to reflect a potential discrepancy in the c.g. location of up to $0.5 \mathrm{~mm}$. When the shock reflection impinges on the body behind the center of gravity $(h / d=0.75,0.5)$, the high-tolow pressure distribution around the boat tail section results in a nosedown moment. The opposite is true at $h / d<0.5$, where the reflected bow shock impinges on the body upstream of the center of gravity, resulting in a nose-up moment that increases significantly in magnitude with decreasing clearance.

The $z$-moment acts on the projectile even in free flight, due to the influence of spin. It is amplified by decreasing ground clearance, which would increase the tendency for the body to yaw, although the magnitude of the moment is small considering that the projectile is axially aligned with the freestream in all cases.

The coupling of a pitching moment, a mild yaw moment, a mild side force and a strong normal force would lead to a complex motion. Although we do not make any estimates as to changes in trajectory here, it can be deduced that the deviation of the projectile from its path if the ground plane were infinite may become significant, and the dynamic stability of the body could be critically affected. However, over any reasonably realistic ground or wall plane distance the influence on its final destination may well prove to be minimal.

\section{Pressure Distributions}

To better understand the forces that act on the projectile in ground effect, the pressure distributions for various clearances are presented in Fig. 24, as coefficients on the axial $z=0\left(0 / 180^{\circ}\right.$ plane $)$ plane and as contours on the projectile surface.

The contours serve to highlight the points of reflection impingement on the projectile, and also the way in which the reflected shocks wrap around the body. At clearances of $h / d=0.365$ and 0.2 , the bow shock reflection interacts with the ogive forebody, where the pressure is already higher than over the rest of the body, and therefore the high pressure spike created by the shock is of considerably greater magnitude. At higher clearances the bow shock reflection is not only naturally weaker but has been influenced by the expansions around the forebody and thus the pressure increase across the shocks is not as
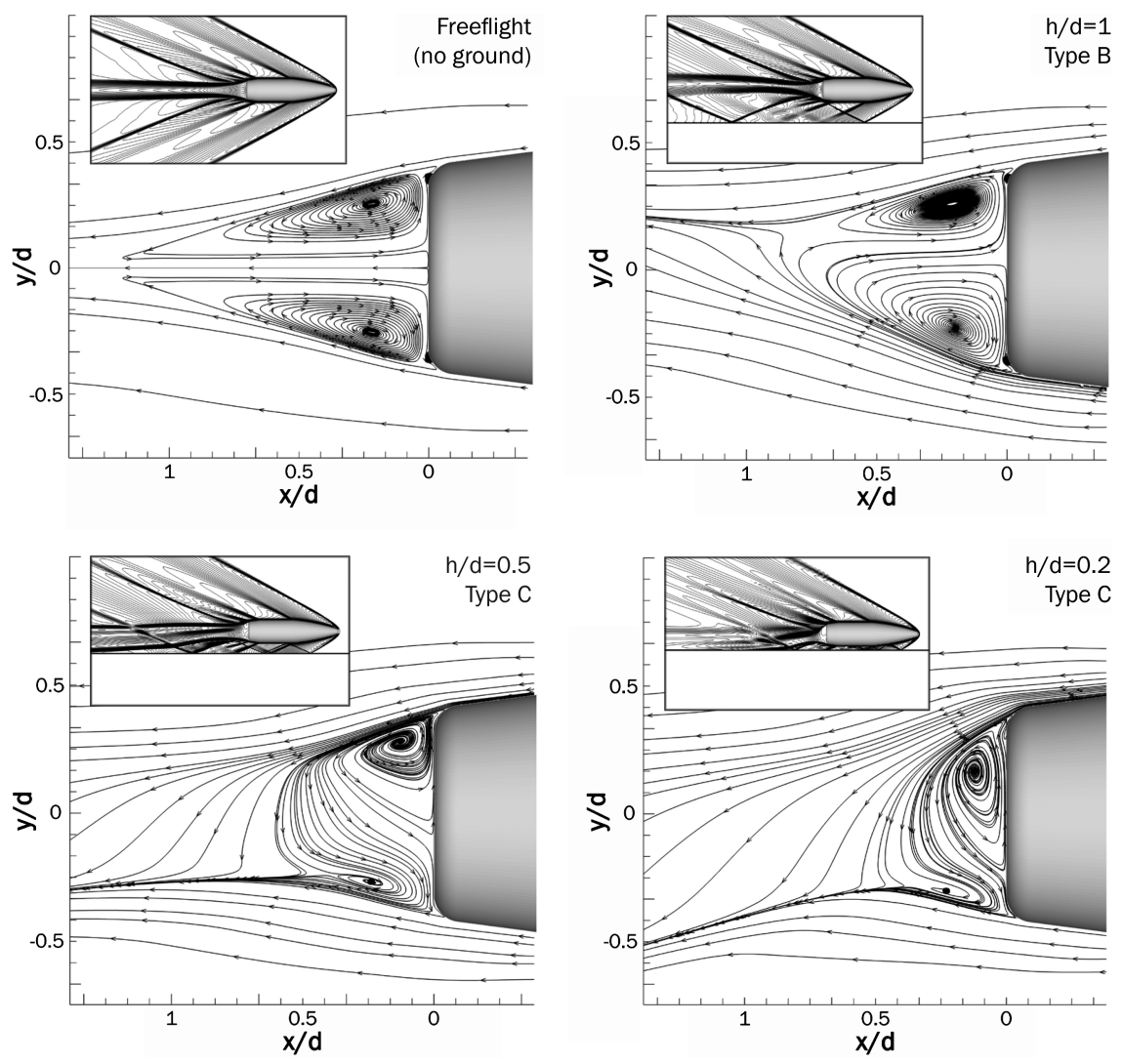

Fig. 25 Streamlines in the near-wake region highlighting the influence of ground clearance on recirculation cells. 
great. The high pressure created behind the reflection impingement for $h / d=0.5$ creates a center of lift that is behind the center of gravity, causing the nose-down pitching moment shown in Fig. 23. This tendency is reversed at lower clearances when one or more shock interactions cause increasing high pressure on the lower side of the body ahead of the c.g.

As ground clearance is reduced, pressure drops over the rear of the projectile. This is due to the reflection of expansion waves (which at higher clearances simply diffuse into the far field) interacting with the rearward portion of the body. This concentration of low pressure, due to a constriction of the propagation of the expansion waves between the projectile and the ground, is the prime contributing factor to the wake deflections observed earlier, and produces the changes to pitching behavior discussed previously.

\section{Flow in the Wake}

The flow mechanisms in the wake are largely responsible for the change in drag shown in Fig. 23. It was previously seen in Fig. 16 that the wake experiences different forms of deflection: an upward deflection for a type $\mathrm{B}$ reflection interaction and eventually a downward deflection for a type $\mathrm{C}$ case.

This is caused by the pressure difference across the area between the projectile and the ground and that above it; pressure rises across the reflected shocks, so the natural tendency of the flow in a type B scenario is to move upwards from the high pressure region to where it is lower, in the expansions above the wake. With closer proximity to the ground comes the concentration of the low pressure caused by the constrained, reflecting expansion waves, such that the area immediately below the wake draws air downwards.
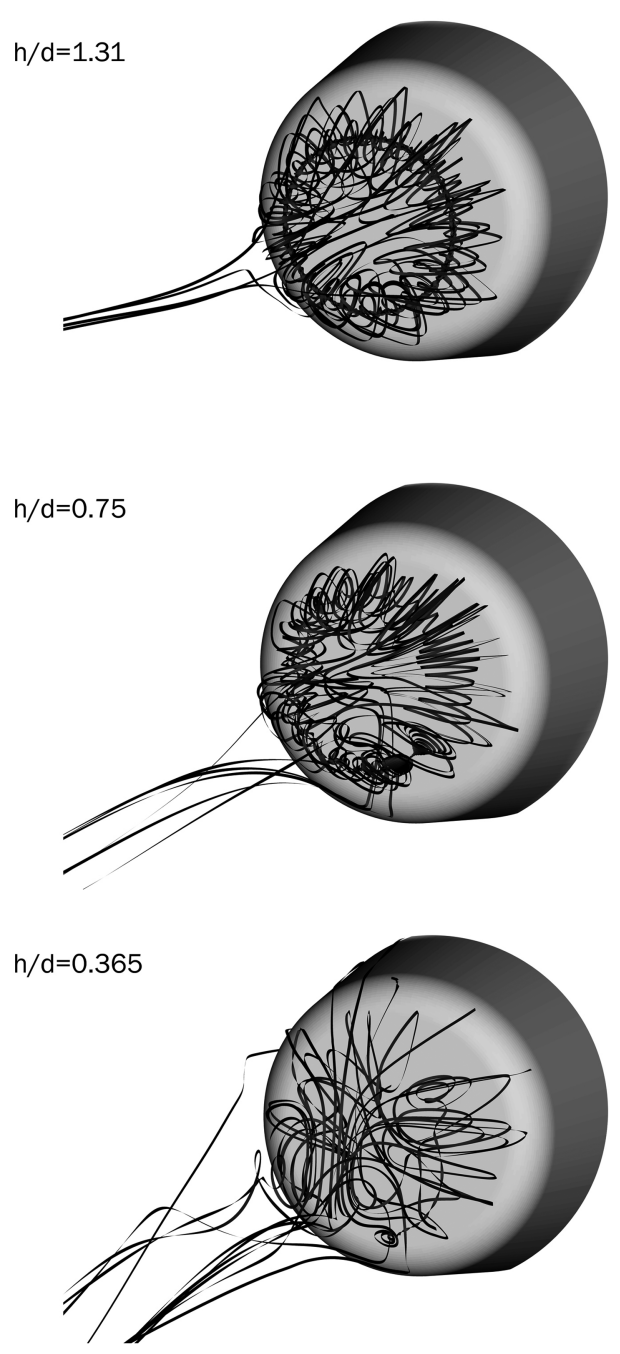

Fig. 26 Path lines released from a plane $1 \mathrm{~mm}$ from the base of the spinning projectile.
The flow structures in the near wake and inside the primary recirculation region are strongly influenced by the changes in pressure distribution in the flowfield around the projectile. Figure 25 presents streamlines on the $0 / 180^{\circ}$ plane at $z=0$ in the base region for various ground clearances. In free flight there are two distinct recirculation cells, perfectly axisymmetric. Also of note are small but distinct separated zones on the blend from the boat tail to the base.

At $h / d=1$, the bow shock reflection interacting with the wake results in the upwards deflection previously discussed, and the upper recirculation cell becomes compressed, as is consistent with the images presented in slices 5 and 6 of Fig. 19 as the wake distorts on all planes. The direction of flow is from the lower cell to the upper one. At $h / d=0.5$, this trend is reversed, again consistent with the deflections noted earlier, as flow now proceeds from the upper cell to the lower one, although both are now highly distorted compared with the regular structures seen in the freestream case. All recirculation is confined to a region within $0.5 d$ of the base.

This trend continues at $h / d=0.2$ where the downwash is at its most extreme, and the recirculation is confined to within $0.3 d$ of the base and is made up of a large upper cell and a weak lower one. The vertical extent of the recirculation has also diminished, and the lower cell has almost ceased to exist. The shear layer on the lower side angles downwards from $0.5 d$ of the base, such that it soon interacts with the ground.

Although the wake is structurally similar for the no-spin and spinning projectile, the rotational component introduces a slight axial twist, which is damped downstream. The jetlike feature previously noted in slices $6-9$ of Fig. 21 is present in both spinning and nonspinning projectile wakes, as fluid from above is entrained
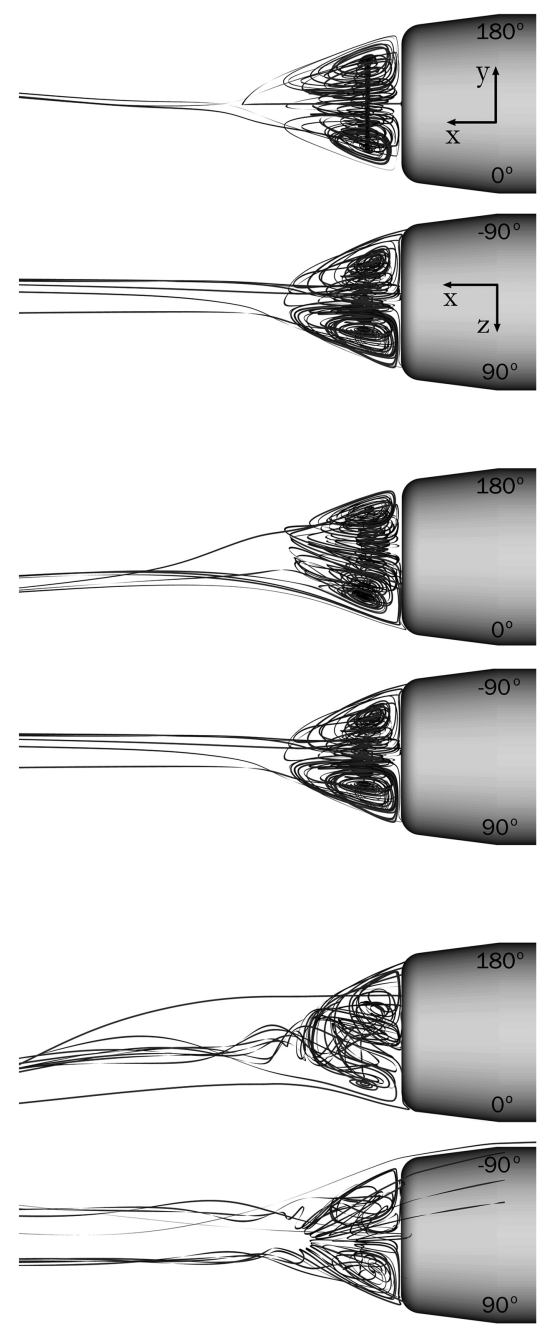
into the counter-rotating cells that make up the vortex cores of the wake. This accounts for the thickening of the wake even in the absence of disruptive wave interactions, as the wake expands. At low clearances, the wake structures eventually interact with the ground plane, and the vortices begin to spread laterally.

It is interesting to note that the wake patterns and the more general wake deflections discussed throughout the text are reminiscent of the results of base flow studies in literature on military projectiles at angles of incidence from 2 to $5^{\circ}[6,26,27]$.

Looking again at the recirculation region behind the full spinning projectile, now in a three-dimensional context, the effect of ground proximity on the near-wake region reveals additional flow structures not apparent from the planes already examined in Fig. 25 . Figure 26 presents path lines, released from five points, $1 \mathrm{~mm}$ from the base; four spaced equally at a radial distance of $2 \mathrm{~mm}$ from the projectile axis of revolution, and one on the axis itself.

The way in which the recirculation structures break down is well illustrated in the three-dimensional representation. At $h d=1.31$ the immediate wake is well structured, with a strong rotating vortex central to the recirculations observed on the planar diagrams. At $h / d=0.75$, the structures begin to break down: the circular vortex has distorted and forms a much looser helix. It also appears to feature a cyclonic component in the lower right quadrant. This feature is also present at $h / d=0.365$, although by now the streamlines indicate a severe breakdown of the regimented recirculation structures in the vertical plane, completing a trend of transition from a largelysymmetric, ordered structure to a highly asymmetric, more incoherent flow. The wake deflections observed earlier can also be noted in the streamlines, with the type B case exhibiting a strong upwards deflection following the shock interaction, while the streamlines for the type $\mathrm{C}$ cases increasingly deflect downwards with closer ground proximity.

\section{Conclusions}

A wide range of ground clearances were investigated for both spinning and nonspinning projectiles to establish changes to the flowfield around the projectile, and to determine both their causes and effects.

Three types of shock reflection interactions with the projectile in ground effect were identified based on this study. A type A interaction $(h / d>1.31)$, whereby the reflection of the projectile bow shock from the ground plane impinged only on the far wake, was found to exert little influence on the aerodynamic forces and moments of the body. A type B case $(1 \leq h / d \leq 1.31)$ was defined as featuring a strong interaction between the reflected bow wave and the near wake of the projectile, at which point the aerodynamic forces and moments were found to experience a change from freestream conditions, with drag decreasing. A type $\mathrm{C}$ case $(h / d \leq 0.75)$ produced a strong normal force acting on the projectile, which, due to the body's rate of spin, would produce a lateral precession. Drag increased significantly as the wake was thickened and drawn toward the ground, and a weak lateral force was also discovered as ground clearance was reduced, which, again as the projectile is spinning, would produce a small influence on the vertical movement of the projectile relative to the ground plane.

The projectile was discovered to experience a marked pitching moment, initially nose-downwards at the high end of type $\mathrm{C}$ reflection interactions, then more strongly nose-up with continued decreasing ground clearance, as the pressure distribution over the rear of the projectile was increasingly distorted by the build up of high pressure behind the series of shock/ground interactions.

Reflections downstream of the projectile interacted heavily on the wake, distorting its structure and drawing it initially up (type A and B cases), then strongly downwards to interact with the ground in the far field (lower type $\mathrm{C}$ cases). These interactions were shown to be complex and highly three-dimensional, and CFD provided a wealth of information that was not gleaned from the original live-range or wind-tunnel experiments: the detailed wake profiles, wake flow structures and the reason for their deflections, the ground pressure distributions describing the "footprint" of the shocks there, visualization of the propagation of the shocks and their reflections in three dimensions, and force and moment data.

\section{References}

[1] Cranz, C., Lehrbuch der Ballistik, Springer, Berlin, 1925, pp. 124-179.

[2] Purdon, J. P., "Supersonic Projectiles in the Vicinity of Solid Obstacles," Undergraduate Thesis, Univ. of New South Wales at the Australian Defence Force Academy, Canberra, Australia, 2006.

[3] Purdon, J. P., Mudford, N. R., and Kleine, H., "Supersonic Projectiles in the Vicinity of Solid Obstacles," Proceedings of the 27th International Congress of High-Speed Photography and Photonics, Vol. 6279, SPIE, Bellingham, 2007, pp. 1-8.

[4] Doig, G., Barber, T. J., Leonardi, E., Neely, A. J., and Kleine, H., "Methods for Investigating Supersonic Ground Effect in a Blowdown Wind Tunnel," Shock Waves, Vol. 18, No. 2, 2008, pp. 155-159. doi:10.1007/s00193-008-0144-z

[5] Barber, T. J., Leonardi, E., and Archer, R. D., "Causes for Discrepancies in Ground Effect Analyses," Aeronautical Journal, Vol. 106, No. 1066, 2002, pp. 653-667.

[6] Silton, S. I., "Navier-Stokes Computations for a Spinning Projectile from Subsonic to Supersonic Speeds," Journal of Spacecraft and Rockets, Vol. 42, No. 2, 2005, pp. 223-231.

doi: $10.2514 / 1.4175$

[7] Catalano, G. D., and Sturek, W. B., "A Numerical Investigation of Subsonic and Supersonic Flow Around Axisymmetric Bodies," U.S. Army Research Lab. Rept. ARL-TR-2595, 2001.

[8] Silton, S. I., and Webb, D. W., "Experimental Determination of the Effect of Rifling Grooves on the Aerodynamics of Small Caliber Projectiles," AIAA Atmospheric Flight Mechanics Conference and Exhibit, Keystone, CO, 21-24 Aug. 2006.

[9] Weinacht, P., "Validation and Prediction of the Effect of Rifling Grooves on Small-Caliber Ammunition Performance," AIAA Atmospheric Flight Mechanics Conference and Exhibit, Keystone, CO, 21-24 Aug. 2006.

[10] Weinacht, P., Sturek, W., and Schiff, L., "Projectile Performance, Stability and Free-Flight Motion Prediction Using Computational Fluid Dynamics," Journal of Spacecraft and Rockets, Vol. 41, No. 2, 2004, pp. 257-263. doi: $10.2514 / 1.1037$

[11] Sahu, J., "Time-Accurate Numerical Prediction of Free-Flight Aerodynamics of a Finned Projectile," AIAA Atmospheric Flight Mechanics Conference and Exhibit, San Francisco, CA, 1518 Aug. 2005.

[12] DeSpirito, J., and Heavey, K., "CFD Computation of Magnus Moment and Roll Damping Moment of a Spinning Projectile," U.S. Army Research Lab. Rept. ARL-RP-131, 2006.

[13] Torda, T. P., and Morel, T. A., "Aerodynamic Design of a Land Speed Record Car," Journal of Aircraft, Vol. 8, No. 12, 1971, pp. 1029-1033. doi: $10.2514 / 3.59201$

[14] Oberoi, R., Chakravarthy, S., Fredrick, W., and Glessner, P. T., "Using CFD to Design the American Challenger Rocket Car," Society of Automotive Engineers Paper 206-013660, 2006.

[15] Noble, R., and Tremayne, D., Thrust, Partridge, London, 1998.

[16] Strike, W. T., and Lucas, E. J., "Evaluation of Wind Tunnel Tests on AFMDC Monorail Cone and Spike Nose Sled Configurations at Mach Numbers from 2.0 to 5.0," AEDC-TR-68-198 (AD679206), Arnold Engineering Development Center, TN, 1968.

[17] Rhudy, R. H., and Corce, J. D., "Static Force and Moments Tests of the Holloman Narrow-Gage Rocket Sled at Mach Numbers from 1.5 to 4," AEDC-TR-75-29 (ADA014259), Arnold Engineering Development Center, TN, 1975.

[18] Marconi, F., "Shock Reflection Transition in 3-Dimensional Steady Flow About Interfering Bodies," AIAA Journal, Vol. 21, No. 5, 1983, pp. 707-713. doi: $10.2514 / 3.8137$

[19] "Fluent 6/.3 User's Guide, Vol. 2," Fluent, Inc., Lebanon, NH, 2006.

[20] DeSpirito, J., Silton, S. I., and Weinacht, P., "Navier-Stokes Predictions of Dynamic Stability Derivatives: Evaluation of Steady State Methods," U.S. Army Research Lab. Rept. ARL-TR-4605, 2008.

[21] Sturek, W. B., Nietubicz, C. J., Sahu, J., and Weinacht, P. J., "Applications of Computational Fluid Dynamics to the Aerodynamics of Army Projectiles," Journal of Spacecraft and Rockets, Vol. 31, No. 2, 1994, pp. 186-199. doi: $10.2514 / 3.26422$

[22] Spalart, P., and Allmaras, S., "A One-Equation Turbulence Model for Aerodynamic Flows," Recherche Aérospatiale, Vol. 1, No. 1, 1994, pp. 5-21. 
[23] Shih, T. H., Liou, W. W., Shabbir, A., Yang, Z., and Zhu, J., "A New $k-\varepsilon$ Eddy-Viscosity Model for High Reynolds Number Turbulent Flows: Model Development and Validation," Computers and Fluids, Vol. 24, No. 3, 1995, pp. 227-238. doi:10.1016/0045-7930(94)00032-T

[24] Menter, F. R., "Two-Equation Eddy-Viscosity Turbulence Models for Engineering Applications," AIAA Journal, Vol. 32, No. 8, 1994, pp. $1598-1605$.

[25] Rusanov, V. V., "A Blunt Body in a Supersonic Stream," Annual Review of Fluid Mechanics, Vol. 8, 1976, pp. 377-404. doi:10.1146/annurev.fl.08.010176.002113
[26] Sahu, J., "Numerical Computations of Supersonic Base Flow with Special Emphasis on Turbulence Modeling," AIAA Journal, Vol. 32, No. 7, 1994, pp. 1547-1549. doi: $10.2514 / 3.48296$

[27] Sahu, J., "Numerical Computations of Transonic Critical Aerodynamic Behavior," AIAA Journal, Vol. 28, No. 5, 1990, pp. 807-816. doi: $10.2514 / 3.25123$

F. Coton Associate Editor 